\title{
Dynamics of small-scale magnetic fields on the Sun: observations and numerical simulations ${ }^{\star}$
}

\author{
N. Bello González ${ }^{1}$, L. Yelles Chaouche ${ }^{2}$, O. Okunev ${ }^{1,3}$, and F. Kneer ${ }^{1}$ \\ 1 Institut für Astrophysik, Friedrich-Hund-Platz 1, 37077 Göttingen, Germany \\ e-mail: [nazaret, olok, kneer] @astro.physik. uni-goettingen.de \\ 2 Max-Planck-Institut für Sonnensystemforschung, Max-Planck-Str. 2, 37191 Katlenburg-Lindau, Germany \\ e-mail: yelles@mps.mpg.de \\ 3 Central Astronomical Observatory of the Russian Academy of Sciences, Pulkovskoye chaussee 65/1, 196140 St. Petersburg, Russia
}

Received 23 June 2008 / Accepted 18 September 2008

\section{ABSTRACT}

\begin{abstract}
Context. Small-scale magnetic fields play an important role in the structure and the dynamics of the solar atmosphere. Aims. This study aims at revealing the evolution of magnetic fields, together with granular convection in the quiet Sun by means of observations with high spatial, spectral, and temporal resolution and of numerical MHD simulations.

Methods. Time sequences from quiet Sun disc centre were obtained with the upgraded "Göttingen" Fabry-Perot spectropolarimeter at the Vacuum Tower Telescope, Observatorio del Teide/Tenerife, in the Fe I 6173 Å line. The data were reconstructed with speckle methods. For comparison with the observations, numerical simulations of granular magnetoconvection were carried out with the MURaM code. The intensities and Stokes vectors emerging from the simulation box were degraded in wavelength, spatial co-ordinates, and noise to the quality of the observations.

Results. The noise in the observed magnetograms from the centre-of-gravity method is $\sigma_{B} \approx 2 \mathrm{G}$, yielding a polarimetric sensitivity of $3 \times 10^{15} \mathrm{Mx}$, at a cadence of $23 \mathrm{~s}$ with $0^{\prime}$ '33 spatial resolution in a field of view of $\sim 33^{\prime \prime} \times 25^{\prime \prime}$. Many of the observed $V$ profiles in network and internetwork (IN) areas exhibit strong asymmetries that indicate strong magnetoconvection. The temporal evolutions of IN structures and of a bright point (BP), as seen in broadband and line-minimum images, in Dopplergrams, and in magnetograms, are presented. The magnetic field structure in the numerical MHD simulations is even more complex than seen in the observations. Correspondingly, the emergent Stokes profiles are often very abnormal. The degradation yields a reduction of the intrinsic field strength to the "observed" one by a factor 4-5. The spectral resolution of the spectrometer is adequate, yet the limitation in spatial resolution and by noise filtering swamps the details seen in the non-degraded simulations. A BP was not found in the simulations, presumably because BP's are not so common and the simulated box is quiet small: only $6 \mathrm{Mm}$ wide in both horizontal directions. Conclusions. The combination of high-resolution observations with numerical simulation is a highly valuable means for studying small-scale magnetic fields on the Sun. Two-dimensional, low-noise data with spectral resolution as good as achieved here and with spatial resolution of $0 !^{\prime} 1$ and better are needed to better understand this important part of the solar magnetism.
\end{abstract}

Key words. Sun: magnetic fields - techniques: high angular resolution - techniques: polarimetric - methods: numerical - methods: observational

\section{Introduction}

Small-scale fields on the Sun and their dynamics have become a lively field of research during the past decades. Since the discovery of "magnetic knots" by Beckers \& Schröter (1968) and of kilo-Gauss fields in the network of the quiet Sun by Stenflo (1973), steady improvement in polarimetric sensitivity and spatial resolution has revealed that the internetwork (IN) magnetic fields play an important role in the solar magnetism. Lites et al. (1996) estimated the IN flux emergence to $2.6 \times$ $10^{24} \mathrm{Mx} /$ day, which exceeds the estimated flux emerging in active regions and ephemeral magnetic regions of $\sim 10^{20} \mathrm{Mx} /$ day and $\sim 10^{22} \mathrm{Mx} /$ day, respectively, by orders of magnitude (cf. Durney et al. 1993; Petrovay \& Szakály 1993).

The literature on results from ground-based observations of IN fields is rich (e.g., Keller \& et al. 1994; Lin \& Rimmele 1999; Lites et al. 1999; Domínguez Cerdeña et al. 2003; Khomenko et al. 2003; Socas-Navarro et al. 2004; Martínez González et al. 2008, and references therein). Recently, the high-resolution

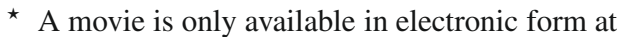
http://www. aanda.org polarimetric data obtained with the Solar Optical Telescope (SOT; e.g. Suematsu et al. 2008) on board the HINODE satellite have uncovered a wealth of small-scale magnetic structures, both in active and in quiet regions, also of IN fields (e.g. Centeno et al. 2007; Orozco Suárez et al. 2007; Rezaei et al. 2007; Lites et al. 2008). While the above measurements of small magnetic flux are based on analyses using the Zeeman effect, the Hanle effect allowed important findings on weak and entangled, turbulent fields, which are hidden to Zeeman diagnostics (e.g. Bianda et al. 1999; Faurobert et al. 2001; Trujillo Bueno et al. 2004).

On the side of numerical simulations of small-scale magnetic field dynamics, huge progress has been achieved by several groups during the last years. Magnetohydrodynamic (MHD) simulations of magnetoconvection have arrived at a high degree of realism on small scales in space and time (Vögler et al. 2005; Stein \& Nordlund 2006; Abbett 2007, see also the review by Steiner 2007). The spatial scales resolved in the simulations are typically around $20 \mathrm{~km}$, much smaller than the spatial resolution of $150-360 \mathrm{~km}(\sim 0.2-0.5)$ obtained in today's observations of magnetic fields. Thanks to their superb resolution, much can be learned from the MHD simulations by 
Table 1. Observing parameters. The $r_{0}$ is Fried parameter estimated by KAOS and $\delta \lambda$ the scanning step.

\begin{tabular}{cccccccc}
\hline \hline Date & $r_{0}[\mathrm{~cm}]$ & $\delta \lambda[\mathrm{m} \AA]$ & $t_{\text {exp }}[\mathrm{ms}]$ & \# Cycles & \#Sp. Pos. & $\Delta t[\mathrm{~s}]$ & \# Scans \\
\hline 23.07 .07 & $14-23$ & 17.7 & 15 & 8 & 30 & $\sim 23$ & 13 \\
25.07 .07 & $9-25$ & 17.7 & 15 & 8 & 27 & $\sim 43$ & 32 \\
\hline
\end{tabular}

comparison with observations. E.g., Janßen et al. (2003) studied fractal dimensions of facular regions with observation and simulation. Khomenko et al. (2005a,b) used MHD simulations of granular magnetoconvection for an interpretation of observations of IN magnetic fields in visible and infrared spectral lines. De Pontieu et al. (2006) compared MHD modelling with highresolution observations to study the rapid temporal variability of faculae. Schüssler \& Vögler (2008) found horizontal magnetic fields in their simulations in agreement with observations, e.g. from HINODE (Lites et al. 2008). They concluded that a nearsurface turbulent dynamo is a viable mechanism for generating dominating horizontal fields in the mid photosphere (see also Steiner et al. 2008).

We investigate small-scale magnetic field dynamics on the Sun with both high-resolution observations and MHD simulations. We observed quiet Sun and weak activity with the two-dimensional Göttingen Fabry-Perot interferometric spectropolarimeter (in short FPI) at the Vacuum Tower Telescope (VTT)/Observatorio del Teide/Tenerife (Bendlin et al. 1992; Bendlin \& Volkmer 1995; Volkmer et al. 1995; Koschinsky et al. 2001). The upgrades of this instrument in 2005 (Puschmann et al. 2006) and in 2007 (Bello González \& Kneer 2008) had been successful. The performance of the FPI in spatial resolution ( 0.25 in broadband images and 0.'33 in magnetograms) and spectral resolution $(\sim 25 \mathrm{~m} \AA)$ is as good as that of the SOT spectrometer/polarimeter on board of HINODE. In the data taken for this work, the polarimetric sensitivity of the FPI is $\sim 2 \times 10^{-3} I_{\mathrm{c}}$ (with $I_{\mathrm{c}}=$ continuum intensity), approximately a factor of two less than the usual HINODE SOT polarimetric data. The temporal resolution with cadences of 20-50 s, however, is much better, thanks to the superior throughput of FPIs (e.g. Kneer 2007). In addition, the computing capacities for MHD simulations have again increased considerably during the past few years. It has become feasible to follow the temporal evolution of magnetic fields in 3D simulations of magnetoconvection for many minutes (of real time on the Sun). This allows us to compare time sequences of observed, small-scale magnetic structures with theoretical expectation and to make predictions on observations with still higher spatial resolution.

This paper is organised as follows. In Sect. 2, we describe the observations and the data analysis. Section 3 presents the observational results. Section 4 deals with the MHD simulations and their degradation for comparison with the observations. Section 5 compares the observations and the simulations, and Sect. 6 concludes the paper.

\section{Observations and data analysis}

\subsection{Observations}

The observations for this study were taken on July 23 and on July 25, 2007, from disc centre of the Sun. We used the Göttingen FPI spectrometer/polarimeter (Puschmann et al. 2006; Bello González \& Kneer 2008) at the VTT/Observatorio del Teide/Tenerife. The observations were supported by the Kiepenheuer Adaptive Optics System (KAOS, von der Lühe et al. 2003). On both days, the Fe I $6173 \AA$ line, with Landé factor $g_{\mathrm{L}}=2.5$, was scanned with the spectrometer. The reduction of the area to be read out from the CCDs allowed reduction of the scanning time. The field of view (FOV) is still appreciable, $\sim 33^{\prime \prime} \times 25^{\prime \prime}$, with a pixel size corresponding to 0 ' 109 . Table 1 gives the parameters of the observations.

The polarimeter is based on two ferroelectric liquid crystals (FLCs), a (nominal) $\lambda / 2$ retarder plate followed by a (nominal) $\lambda / 4$ retarder, and a modified Savart plate (for details see Bello González \& Kneer 2008). This allows the measurement of the full Stokes vector with minimised seeing-induced crosstalk. By readjustment of the FLCs before the observing period, the efficiencies of the polarimeter could be improved compared to those during the first tests of the setup in 2007. They were then $\varepsilon_{Q}=0.53, \varepsilon_{U}=0.32$, and $\varepsilon_{V}=0.61$. The instrumental crosstalk by KAOS and the subsequent optics to the FPI spectrometer has been measured as described in Beck et al. (2005a), while the crosstalk from the telescope proper can be taken into account by means of the polarisation model by Beck et al. (2005b). The $I \rightarrow V$ crosstalk was removed by subtracting an appropriate fraction of $I(\lambda)$ from $V(\lambda)$ such that $V(\lambda) \approx 0$ at continuum wavelengths. Averaged over the FOV, $|V(\lambda)|$ was then found to be less than $3 \times 10^{-4} I_{\mathrm{c}}$.

To further shorten the spectral scanning time, we used a polarimetric setup on July 23 that measures just Stokes $I$ and $V$. For this, the $\lambda / 4$ retarder FLC was removed, while in front of the polarimeter a $\lambda / 4$ retarder was mounted with fixed orientation of its fast axis, under $45^{\circ}$ with respect to the zero orientation of the $\lambda / 2$ retarder. This, together with the Savart plate, provides alternating left and right circularly polarised components on either half of the detector. The efficiency was high, $\varepsilon_{V}=0.91$.

For image reconstruction with speckle methods, broadband frames were recorded strictly simultaneously with the narrowband spectropolarimetric frames. Furthermore, dark frames and flat field frames were taken with varying pointing of the telescope and with defocused telescope. Finally, images from a continuum light source (halogen lamp) were obtained to measure the wavelength dependence of the transmission of the spectropolarimeter.

In the present study, we concentrate on the Stokes $I$ and $V$ signals. The degree of linear polarisation from weak fields of the Sun is low for Fe I 6173 and the signals require a special analysis, as demonstrated by Lites et al. (2008). The analysis of the linear polarisation signals and the discussion of horizontal magnetic field components are certainly important for understanding the IN magnetic fields (e.g. Lites et al. 1996; De Pontieu 2002; Martínez González et al. 2007a,b; Centeno et al. 2007; Lites et al. 2008; Abbett 2007; Steiner et al. 2008). Here, we restrict the analysis to circular polarisation and defer the investigation of the measured Stokes $Q$ and $U$ profiles to future work.

\subsection{Data analysis}

Dark corrections and flat fielding were applied. The broadband frames were reconstructed with the "Göttingen" speckle code (de Boer 1996), which uses the spectral ratio method (von der Lühe 1984) and speckle masking (Weigelt 1977). The 


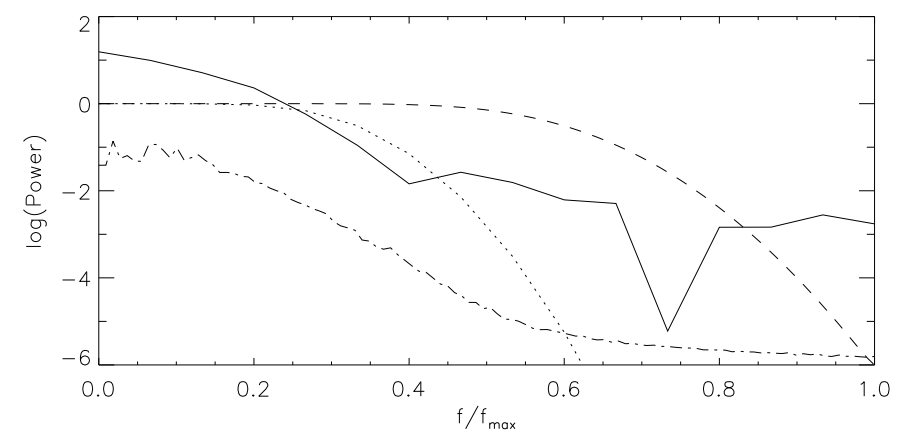

Fig. 1. Power spectrum of $I$ profile from a pixel in the FOV (solid) and azimuthally averaged power spectrum of the continuum intensity fluctuations (dash-dotted), together with their low-pass filters for wavelength (dotted) and for intensity (dashed, radial cut through filter); arbitrary units for power spectra, filters are normalised to 1 at frequency $f=0$; abscissa in units of Nyquist frequency, i.e., $1 /(2 \times$ scanning step width $)$ $=1 /(2 \delta \lambda)$ for wavelength and $1 /(2 \times$ pixel size $)$ for intensity.

speckle reconstruction takes into account that the image correction by KAOS varies with distance from the AO lock point. Thus, the variation in the Fried parameter across the field of view is fitted by a Gaussian centred on the lock point. Such distribution is then used to apply the proper speckle transfer function to each isoplanatic patch (cf. Bello González et al. 2007; Bello González \& Kneer 2008).

Given the estimates of the broadband scenes and the instantaneous speckle images in broad band and narrow band, the speckle reconstruction of the narrow-band images is carried out as in Keller \& von der Lühe (1992) and as repeatedly described in our earlier work (e.g. Krieg et al. 1999; Bello González et al. 2005). The polarimetric measurements of the modulated Stokes vector need special care, as outlined by Bello González \& Kneer (2008). The entangled Stokes signals have to be demodulated, which yields $S_{\lambda}=\left(I_{\lambda}, Q_{\lambda}, U_{\lambda}, V_{\lambda}\right)^{T}$. The Stokes components have then been corrected for instrumental crosstalk.

\subsection{Filtering}

The narrow-band data were noise-filtered in wavelength and in horizontal co-ordinates $(x, y)$ with the filters shown in Fig. 1 . The wavelength filter has a constant value of 1.0 up to a certain frequency, then continues with an exponential decay, and is finally smoothed with a Gaussian. The spatial filter has a transmission of 0.5 at a frequency corresponding to 0.33 .

Finally, after the calculation of the physical parameters (cf. below), their maps were subjected to temporal, subsonic $k-\omega$ filtering ( $k=$ horizontal wavenumber) with a limiting propagation speed of $7.5 \mathrm{~km} \mathrm{~s}^{-1}$.

\subsection{Physical parameters}

We deal with time sequences of intensity images, Dopplergrams, and magnetograms. The intensities, as proxies for temperature fluctuations, are from (speckle reconstructed) broadband images, from images in the continuum $I_{\mathrm{c}}$ near the Fe I line, and from $I_{\min }$ at line minimum. The lattest were determined from parabolic fits from the $I$ profiles at five wavelength points around the minimum. This calculation also yields the velocity $v_{\min }$ from the Doppler shift of the fitted parabola. Furthermore, the centreof-gravity (COG) of the I profiles gives another measurement of the velocity. We calculated the wavelength positions of the COG of profiles with three limits of upper intensity $I_{\mathrm{L}}$ in the profiles for the integration over the profile, $\int\left(\left(I_{\mathrm{L}}-I_{\lambda}\right) / I_{\mathrm{c}}\right) \lambda \mathrm{d} \lambda / \int\left(\left(I_{\mathrm{L}}-\right.\right.$ $\left.\left.I_{\lambda}\right) / I_{\mathrm{c}}\right) \mathrm{d} \lambda$ such that $\left(I_{L}-I_{\min }\right) / I_{\mathrm{c}} \leq a$, with $a=0.9,0.8$, and 0.5 . These will be denoted below by $90 \%, 80 \%$, and $50 \%$ levels, respectively.

We also measured the magnetic fields with the COG method applied to the $\frac{1}{2}\left(I_{\lambda}+V_{\lambda}\right)$ and $\frac{1}{2}\left(I_{\lambda}-V_{\lambda}\right)$ profiles (Semel 1967; Rees \& Semel 1979). According to these authors, this yields the line-of-sight (LOS) components of the magnetic fields averaged over the resolution element and over the formation height, and reduced by spurious light from scattering in Earth's atmosphere and by the telescope optics. We denote this as

$B_{\mathrm{LOS}}=\alpha \overline{\cos \gamma|\boldsymbol{B}|}$,

where $\gamma$ is the angle between the magnetic field $\boldsymbol{B}$ and the LOS, " $\ldots$ " denotes the averaging, and $\alpha<1$ accounts for the spurious light.

The zero position in the wavelength axes of the $V$ profiles presented below are shifted by $+8.6 \mathrm{~m} \AA$ relative to the COG position of the average $I$ profiles in the FOVs (from disc centre). This corresponds to the convective blueshift of the average $I$ profile due to granular motion (Schröter 1957). As will be shown and discussed below, the $V$ profiles become very asymmetric at high spatial resolution in both the observations and the simulations. Sometimes, only one $V$ lobe is present, sometimes two positive/negative lobes, strongly shifted in wavelength (see also Sigwarth et al. 1999). We thus refrain from trying inversions of such profiles. We do not feel confident that a Milne-Eddington inversion assuming magnetic field and velocity independent of height, or a two-component inversion with one magnetic and one non-magnetic component, will lead to realistic descriptions. Recent work by Socas-Navarro et al. (2008) has cast doubts on the reliability of inversions from line pair observations to determine the field strengths. We compare the observed profiles and the physical parameters derived from them with those from simulated model atmospheres.

To obtain an idea of the formation height of the signals, we calculated response functions for velocity $R F_{\text {vel }}$ and magnetic fields $R F_{\text {mag }}$ in the VALC model atmosphere (Vernazza et al. 1981). The following atomic parameters for the Fe I $6173 \AA$ line were chosen: statistical weight of lower level $g=3$, oscillator strength $f=4.4 \times 10^{-4}$, and abundance of iron $\varepsilon_{\mathrm{Fe}}=2.7 \times 10^{-5}$. We employed the method by Eibe et al. (2001). There, small perturbations are introduced as step functions that are $>0$ and constant from bottom to a specific height and zero above that height. Differences in the emergent intensities, or COG values, from varying height of the step, yield the response functions. The perturbation amplitudes were chosen as $0.25 \mathrm{~km} \mathrm{~s}^{-1}$ in the case of velocities and $250 \mathrm{G}$ for magnetic fields. Figure 2 shows the response functions $R F_{\mathrm{vel}}$ and $R F_{\mathrm{mag}}$, for various limits of the profile levels included in the determination of $v$ and $B_{\mathrm{LOS}}$ with the COG method. Similar to Wunnenberg et al. (2002), we obtain a better height discrimination of the formation of the velocity signal by the linear combinations

$R F_{\text {vel,low }}=\left(R F_{\text {vel }, 90}-0.5 \cdot R F_{\text {vel,min }}\right) / 0.5$
$R F_{\text {vel, high }}=\left(R F_{\text {vel,min }}-0.3 \cdot R F_{\text {vel, } 90}\right) / 0.7$

Here, the subscripts " 90 " and "min" refer to the velocity determined with the limit at the $90 \%$ level and to the velocity from the line minimum shift, respectively, and the subscripts "high" and "low" refer to formation heights at high and low atmospheric layers, respectively. Such combinations with the same coefficients were also applied to the data. As seen from Fig. 2a, 

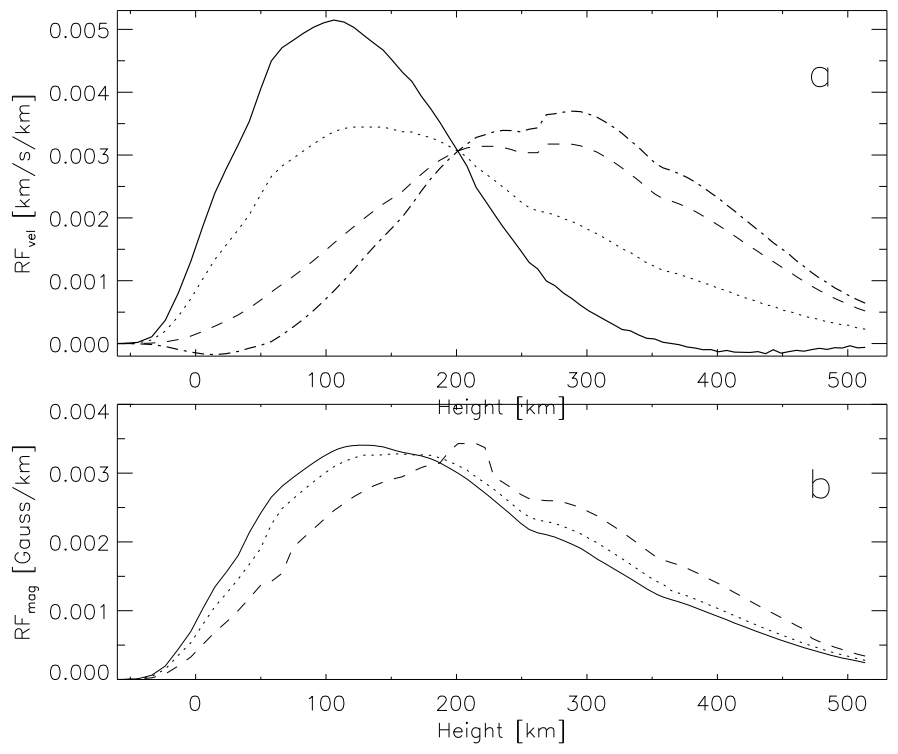

Fig. 2. Upper panel a): velocity response functions $R F_{\text {vel }}$ for $\mathrm{Fe} \mathrm{I}$ $6173 \AA$ A line in VAL C solar atmospheric model (Vernazza et al. 1981). Dotted: $R F_{\text {vel }}$ calculated from COG with intensities below the $90 \%$ level of the $I$ profile (see text for explanation); dashed: from shift of line minimum; solid: linear combination of dotted and dashed according to Eq. (2), giving $R F_{\text {vel,low }}$; dash-dotted: linear combination according to Eq. (3), giving $R F_{\text {vel,high }}$; lower panel $\mathbf{b}$ ): magnetic field response functions $R F_{\text {vel }}$ for Fe I $6173 \AA$ in VALC; solid, dotted and dashed for calculation from COG of $\frac{1}{2}\left(I_{\lambda}+V \lambda\right)$ and $\frac{1}{2}\left(I_{\lambda}-V \lambda\right)$ with intensities below the $90 \%, 80 \%$, and $50 \%$ levels of the profiles, respectively.

the low layer response function $R F_{\text {vel,low }}$ measures velocities at heights of approximately $100 \mathrm{~km}$ (above $\tau_{5}=1$ ), while the high layer response function $R F_{\text {vel,high }}$ measures at heights of approximately $300 \mathrm{~km}$. A similar combination for formation height of the magnetic signal is not meaningful, as seen from Fig. 2b. We thus discuss here only results from the magnetic field determination using profile intensities below the $80 \%$ level for the COG method. This gives information about the field strengths in the height range $50-300 \mathrm{~km}$.

We note that the temperature response function for $\mathrm{Fe} I$ $6173 \AA$ in the VAL C model has its COG at a height of $\sim 270 \mathrm{~km}$ and an $F W H M$ of $\sim 300 \mathrm{~km}$. The line minimum optical depth $\tau_{\min }=1$ is also located at approximately $270 \mathrm{~km}$.

\subsection{Noise}

After filtering we obtained a standard deviation $\sigma=2 \times 10^{-3} I_{\mathrm{c}}$ in the continuum of the $V$ profiles. We added Gaussian noise at each spatial and wavelength position of the proper amount and proportional to $I_{\lambda}$. With two different realisations of noise, this yielded test data $\frac{1}{2}\left(I_{\lambda}+V_{\lambda}\right)$ and $\frac{1}{2}\left(I_{\lambda}-V_{\lambda}\right)$ with the same noise amplitude in the continuum of $V$, at each point in the FOV. The profiles from inter-granular lanes are somewhat more sensitive to noise because they tend to be broader and shallower. However, after applying the above subsonic filter to the noise data, the granular pattern was no longer detected in the "magnetograms". The analysis of the noise-simulated data gave a standard deviation of the field strength of $\sigma_{B} \approx 2.0 \mathrm{G}$. At a $3 \sigma$ significance level and with a spatial resolution of 0.33 , i.e. a radius of the resolution element $r=0.5 \times 725 \times 10^{5} \times 0.33 \mathrm{~cm}$, this translates into a magnetic sensitivity of $3 \sigma_{B} \pi r^{2} \approx 3 \times 10^{15} \mathrm{Mx}$. We note that this sensitivity at high spatial resolution is achieved within $23 \mathrm{~s}$ in an FOV of $\sim 33^{\prime \prime} \times 25^{\prime \prime}$.

\section{Observational results}

We present in Fig. 3 maps from one of the time sequences (2007 July 23): broadband and line minimum images, magnetograms with original amplitude, as well as clipped to $\left|B_{\mathrm{LOS}}\right|=50 \mathrm{G}$, and velocity maps in high and low layers (cf. Eqs. (2) and (3)). In the magnetograms, we show the field strengths multiplied by -1 for presentation purpose. The maps cover, in the upper left quadrant, part of an enhanced network region, as seen from the magnetograms, from the line minimum image, and from the abnormal granulation there. The lower half of the maps appears quiet, with some weak network in the right part and a very quiet internetwork in the lower left $10^{\prime \prime} \times 10^{\prime \prime}$ area. The smallest structures in the magnetograms exhibit an FWHM of 0.'33, which is the spatial resolution of the spectropolarimetric data (see also Bello González \& Kneer 2008).

At the positions with the co-ordinates $(x, y)=\left(15^{\prime \prime} .5,9.3\right)$ and $\left(20 .{ }^{\prime} 8,122^{\prime \prime} 5\right)$, two especially bright points are present in the broadband image, which are also recognised in the line minimum image. We study the temporal evolution of the upper right one below. Apart from the bright points, the FOV is rich in smallscale magnetodynamic processes, and more examples of temporal developments of magnetic finestructures are presented in a separate contribution (Bello González et al. 2008).

In the Dopplergrams, the signal from the 5-min oscillations has been suppressed by subtracting velocity images that were boxcar-smoothed over 3 .' $8 \times 3$ ". 8 . The velocity patterns from the upper and lower atmospheric layers clearly appear different. At high layers, the granular flow structures are roundish, while, in deep layers, they appear flat in the granules with narrow lanes of downflow in the inter-granular lanes. Such structure, with smooth upflow and narrow downflow channels had been found in numerical simulations by e.g. Nordlund et al. (1997). The measured amplitudes in deep layers are high, up to $2.8 \mathrm{~km} \mathrm{~s}^{-1}$. The rms velocities in the deep and high layers amount to $0.59 \mathrm{~km} \mathrm{~s}^{-1}$ and $0.47 \mathrm{~km} \mathrm{~s}^{-1}$, respectively, after subtraction of the 5-min signal.

\subsection{Enhanced network and strong fields}

The enhanced network region in the upper left corner of Fig. 3 possesses strong fields, we measure close to kilo-Gauss field strengths. The magnetogram, in which the fields are clipped to $\left|B_{\mathrm{LOS}}\right|=50 \mathrm{G}$, shows a broadening of the areas with fields $>50$ G. Such expansion was attributed by Lites et al. (1999) to blooming, i.e. scattering of $V$ signal from the strong field areas. We find for the present observations that blooming can only be part of the area broadening. Also, the clipped magnetogram shows finestructure at the borders and outskirts of the strong field areas. In addition, the $V$ profiles change across the "bloomed" areas and can become strongly asymmetric. Four profiles are shown in Fig. 4, two of them exhibiting strong asymmetries. Such asymmetries may occur in the presence of a magnetic canopy (or magnetopause), with rapid changes in magnetic field and velocity along the LOS (cf. Sánchez Almeida et al. 1988; Grossmann-Doerth et al. 1988; Steiner 2000).

\subsection{Temporal evolution of bright point}

The temporal evolution of the bright point structure at $(x, y)=$ $\left(15^{\prime \prime}, 5,9 ! 3\right)$ and its surroundings in Fig. 3 is shown in Fig. 5. The velocities are represented as bright for downward motion and dark for upward motion. The position of the bright point is marked in the five rows by small circles corresponding to 

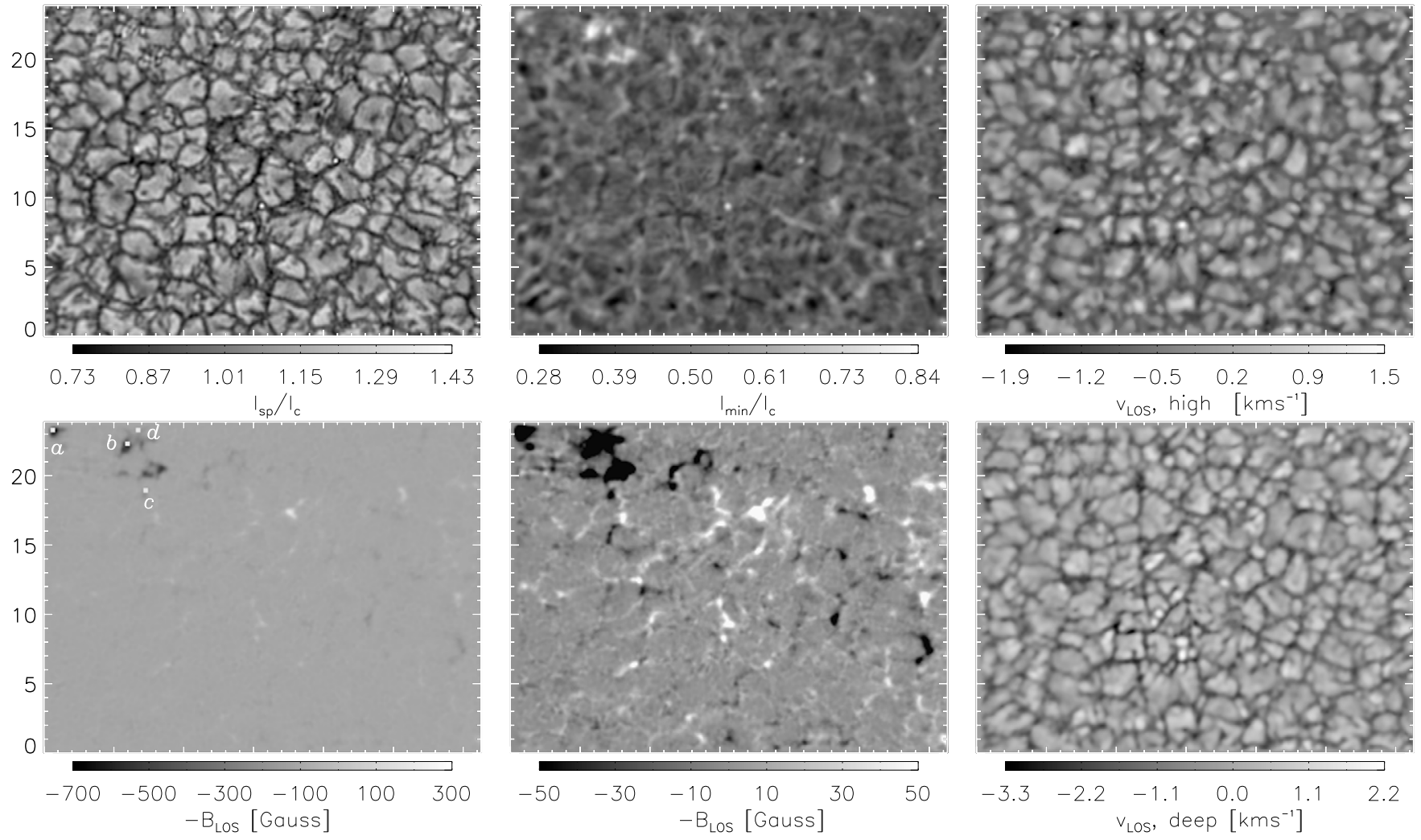

Fig. 3. FOV of the 2007 July 23 time series. Upper left and middle: broadband and line minimum images, respectively, with intensities relative to the average continuum intensity; lower left: magnetogram of $-B_{\mathrm{LOS}}$, minus sign for presentation purpose; lower middle, same magnetogram but clipped to $\left|B_{\mathrm{LOS}}\right|=50 \mathrm{G}$. Upper and lower right panels: velocities in high and deep layers according to Eqs. (2) and (3). The short tick marks are at a distance of $1^{\prime \prime}$. The $V$ profiles from the dots marked with a, b, c, and d are shown in Fig. 4.

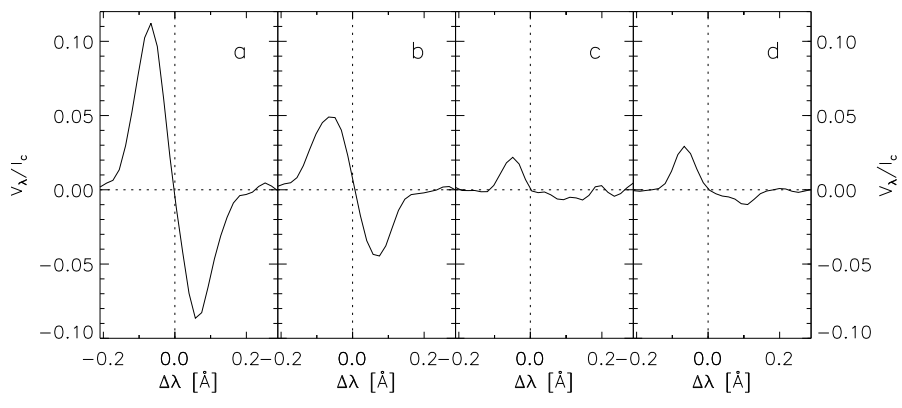

Fig. 4. Examples of $V$ profiles from the enhanced network region in the upper left corner of Fig. 3, labelled there with the same letters as here.

broadband intensities at $1.1 \bar{I}_{\mathrm{bb}}$. The dynamic process took place at the junction of three inter-granular lanes, cf. first row. The granules did not move much during the time sequence and, apparently, did not have any influence on the dynamics, e.g. via squeezing. The bright point in the broadband image attained an intensity of 1.44 of the average intensity in the FOV.

The magnetograms (second row of Fig. 5) exhibit a highly dynamic behaviour. The field structure gets pushed around, splits into parts, disperses, and, at the end of the sequence, has almost dissolved, apparently.

Right at the beginning of the sequence, a small-scale brightening in the line minimum image has started (third row of Fig. 5). Its maximum intensity occurred at approximately the same time as for the bright point in the broadband images.
The velocities from the $I$ profiles (forth and fifth rows of Fig. 5) show downflows in the inter-granular lanes. They are, in high layers, somewhat displaced from the position of the bright point, and coincide better with it at deep layers. Also, the velocities from the $I$ profiles are lower in the upper atmosphere than in deep layers. There they attain speeds downward of $2.4 \mathrm{~km} \mathrm{~s}^{-1}$.

Figure 6 depicts the evolution of the $V$ profiles from the position marked at image Nr. 8 by the circle in the magnetogram sequence. This was the position of the broadband bright point. The $V$ profiles are averaged over $3 \times 3$ pixel, i.e. over the spatial resolution element, and further smoothed in wavelength. The profiles are very broad. At the beginning of the sequence, their zero crossings are redshifted by $\sim 80 \mathrm{~m} \AA$ which corresponds to a downflow velocity of $3.9 \mathrm{~km} \mathrm{~s}^{-1}$. At that time, the COG gives an LOS component of the field strength of $B_{\mathrm{LOS}}=-43 \mathrm{G}$. In the course of the evolution, the red $V$ lobe has decreased and has essentially vanished at time position Nr. 8 (cf. first profile on the left of the bottom row of Fig. 6). At this time, the remaining lobe is strongly broadened. COG gives a small magnetic field strength of $B_{\mathrm{LOS}} \approx-8 \mathrm{G}$. Assuming that this is indeed the blue lobe, the position where it reaches zero is redshifted by approximately $120 \mathrm{~m} \AA$ corresponding to a downflow velocity of $5.8 \mathrm{~km} \mathrm{~s}^{-1}$. Proceeding in time, this lobe also decreased in amplitude until close to vanishing, $\mathrm{cf}$. the third and second last profiles in the bottom row, and then a "normal", blue-asymmetric $V$ profile, only a little shifted in wavelength apparently started to appear, meaning that the gas in the flux tube had almost come to rest. We again emphasise that the value of $B_{\mathrm{LOS}}$ is calculated by the COG method. If the magnetic structure is unresolved and varies in 


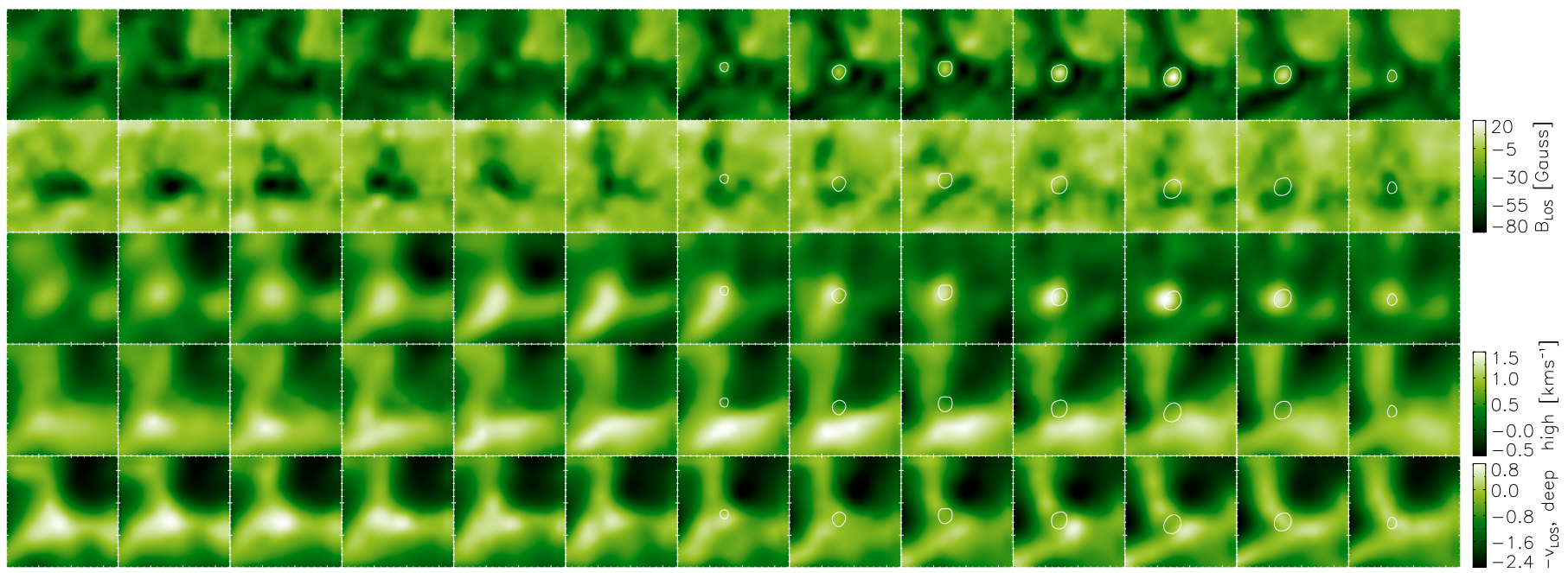

Fig. 5. Temporal evolution of the bright point structure and its surroundings at position $(x, y)=\left(15^{\prime \prime} 5,9\right.$ '. 3$)$ in Fig. 3 . FOV is 1 '.74 $\times 11^{\prime \prime} 74$, time runs from left to right, $\Delta t=23 \mathrm{~s}$; from top to bottom: broadband images, magnetograms, line minimum images, velocities in high and deep layers with bright corresponding to downward motion and dark to upward motion.

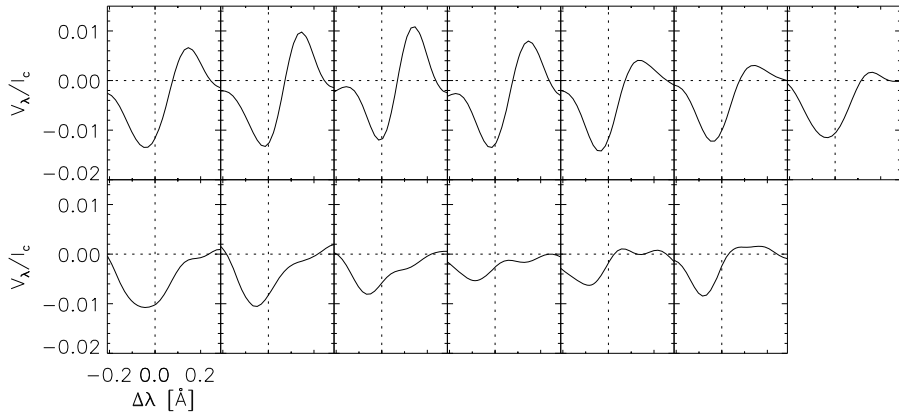

Fig. 6. Temporal evolution of $V$ profiles at the position within the bright point in Fig. 5 where the apparent field decrease occurred. Time runs from left to right, first the upper then the lower row, $\Delta t 23 \mathrm{~s}$, vertical dashed lines are the positions of the COG of $I$ profiles averaged over the FOV plus $8.6 \mathrm{~m} \AA$ (correction for convective blue shift).

brightness with respect to the surrounding non-magnetic plasma then systematic effects in the determination of $B_{\mathrm{LOS}}$ are to be expected.

Changes in the atmospheric states and to the resulting $I, Q$, and $V$ profiles on time scales of $50 \mathrm{~s}$ were obtained in numerical simulations of magnetic flux sheets by Steiner et al. (1998). Nevertheless, the changes in the $V$ profiles in the present study are different from those in the cited simulations. The behaviour of a bright point and of the measured $I$ and $V$ profiles is also different from what is described by Bellot Rubio et al. (2001) and interpreted as convective collapse and upward moving shock. In their observation, the $I$ profiles are strongly blueshifted during the course of the event and a blueshifted $V$ profile arises along the time. In our observations, the $I$ profiles from the bright point are slightly redshifted, if at all, yielding downward velocities of few hundred $\mathrm{m} \mathrm{s}^{-1}$.

Here, we tentatively suggest the scenario that we see a downflow within the magnetic feature caused by a convective collapse (Parker 1979). This has been simulated in the slender flux tube approximation by Hasan \& Schüssler (1985). Their modelling shows that an adiabatic downflow heats high atmospheric layers. The flow velocities in the models are much lower than those measured from the shift in the zero crossing of the two-lobed
$V$ profiles in Fig. 6, though. Also, the bright point studied here is a transient phenomenon. The velocities measured from the $I$ profiles are lower than those measured from the shift of the zero crossing of the $V$ profiles, presumably because the $I$ profiles contain much spurious light from outside the bright point area.

We speculate that the dynamic development comes from the change in the magnetic field structure in the inter-granular lanes and at their junction by subphotospheric magnetoconvection. The magnetograms in Fig. 5 suggest a reorganisation of the magnetic field with splitting, dispersion, and re-enforcement by convective collapse in an unstable situation. The collapse causes an evacuation, consequently a brightening of the broadband intensity originating in the deep interior of the flux structures due to decreased opacity. The brightening in the line minimum images is produced by the downward entrainment of chromospheric gas (Hasan \& Schüssler 1985). The apparent decrease in the magnetic field signal measured with COG results from the heating and evacuation. The magnetic field structure contains little Fe I due to ionisation and strongly reduced gas density. Certainly, this scenario has to be tested by modelling.

The formation of a bright point has also been observed recently by Nagata et al. (2008) with HINODE's SOT. They too report a fast downflow prior to the rise in intensity. From Milne-Eddington inversions, they inferred an increase in the field strength from $500 \mathrm{G}$ to $2000 \mathrm{G}$ during the evolution and concluded with the mechanism of convective collapse. The finally formed magnetic and intensity feature found by Nagata et al. appears to be much more persistent than the bright point discussed here.

\subsection{IN magnetic fields and their temporal evolution}

The IN magnetic fields have attained much attention during the past one to two decades, as outlined in Sect. 1 of the Introduction. We concentrate first on the data from July 23, 2007. In Fig. 7, we show a subfield from a very quiet IN region, the lower left corner in the FOV of Fig. 3 at time position 10 of the sequence, of size $10.9 \times 10^{\prime} 9$. From left to right we present the broadband image, the magnetogram, the line minimum image, and the velocity at deep layers. Comparing the broadband image with the magnetogram shows that the $\left|B_{\mathrm{LOS}}\right|>3 \sigma_{B}$ fields 


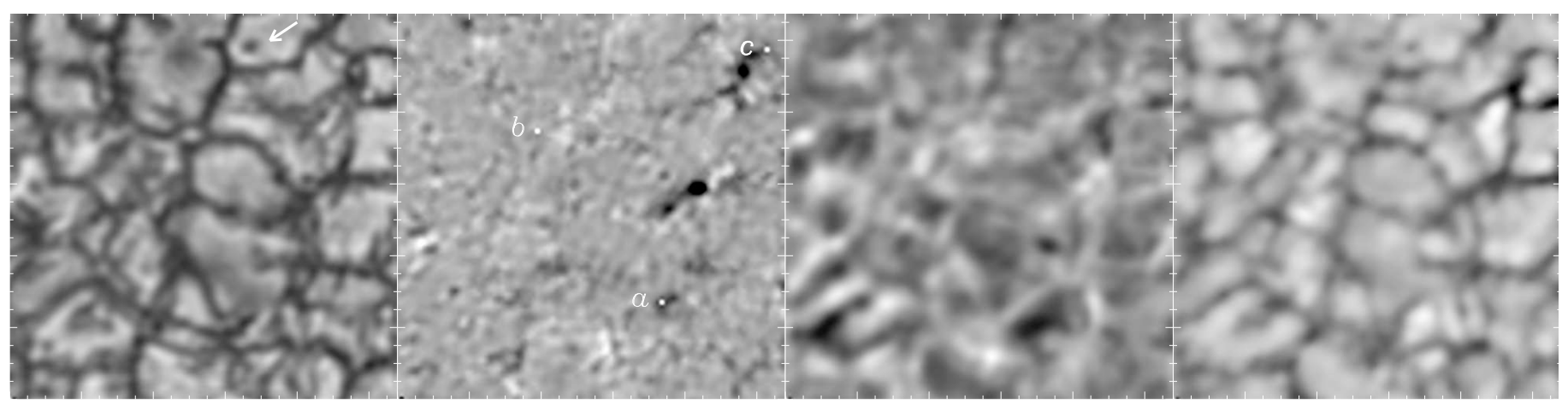

Fig. 7. 10' $9 \times 10^{\prime} 9$ internetwork area from the lower left corner of the FOV in Fig. 3 at time position 10 of the sequence (start at position 0). From left to right: broadband image, magnetogram clipped at $-45 \mathrm{G}<B_{\mathrm{LOS}}<30 \mathrm{G}$, line minimum image, and velocity in deep layers. Short tick marks are at a distance of 0.5 . The arrow points at a dark dot in a granule. The temporal evolution of the $V$ profiles at positions a-c marked with bright dots is shown in Fig. 8.

occur almost exclusively in the inter-granular lanes (see also Bello González \& Kneer 2008, Fig. 8). In a few cases, a weak signal with $B_{\mathrm{LOS}}$ of $10-15 \mathrm{G}$ is detected within granules (cf. Bello González et al. 2008). The line minimum image resembles the inverse granulation pattern, but only partly.

The broadband image shows dark dots within the granules, and an example is indicated by the arrow in Fig. 7. Many more are seen in the broadband image in Fig. 3. Their properties were discussed in detail on the basis of broadband time sequences by Hirzberger et al. (1993). They also are visible in numerical simulations (Vögler et al. 2005; Khomenko et al. 2005b, and Fig. 13 below from the MHD simulations). From an inspection of the temporal evolution of some dark dots we found that they harbour LOS fields of approximately $20 \mathrm{G}$ during their formation (cf. Bello González et al. 2008). Sometimes they disappear while the field remains. Sometimes they develop into intergranular lanes. They exhibit moderate downflows of $0.3-1.0 \mathrm{~km} \mathrm{~s}^{-1}$.

We draw attention to the $V$ profiles in Fig. 8 with lobes that are persistent for several time steps. Tests with pure Gaussian noise of the appropriate amplitude have shown that the shapes of such noisy " $V$ " profiles have amplitudes of $0.002-0.004 I_{\mathrm{c}}$, but only for one time position; i.e., the shapes are not persistent. The changes seen in the $V$ profiles may result both from local development of the magnetic structures and from horizontal motion of the features. We describe the temporal evolution:

- the profiles from position "a" developed from no signal to profiles with one strongly blueshifted lobe and obtained at $t=9$ (second profile from left, lower row), a red lobe. At the end, the profile appeared unshifted with blue asymmetry;

- the profiles in example "b" started with little signal and then became strongly asymmetric with only a red lobe (upper two rightmost and lower two leftmost profiles);

- in example "c", the profile was very red-asymmetric at the beginning, and the blue lobe is not visible at times 1 and 2 (second and third profiles in the upper row). Later from $t=3$ on, an asymmetric three-lobed $V$ profile developed (times 5-7) and became a two-lobed, red-asymmetric profile again (lower row, rightmost profiles).

We emphasise that such asymmetric $V$ profiles are quite common in IN magnetic features, indicating the highly dynamic processes in granular magnetoconvection. We also recall that the COG method, as an averaging method, cannot give detailed information about the magnetic structure and atmospheric dynamics. Probably, inversions on the basis of one-component
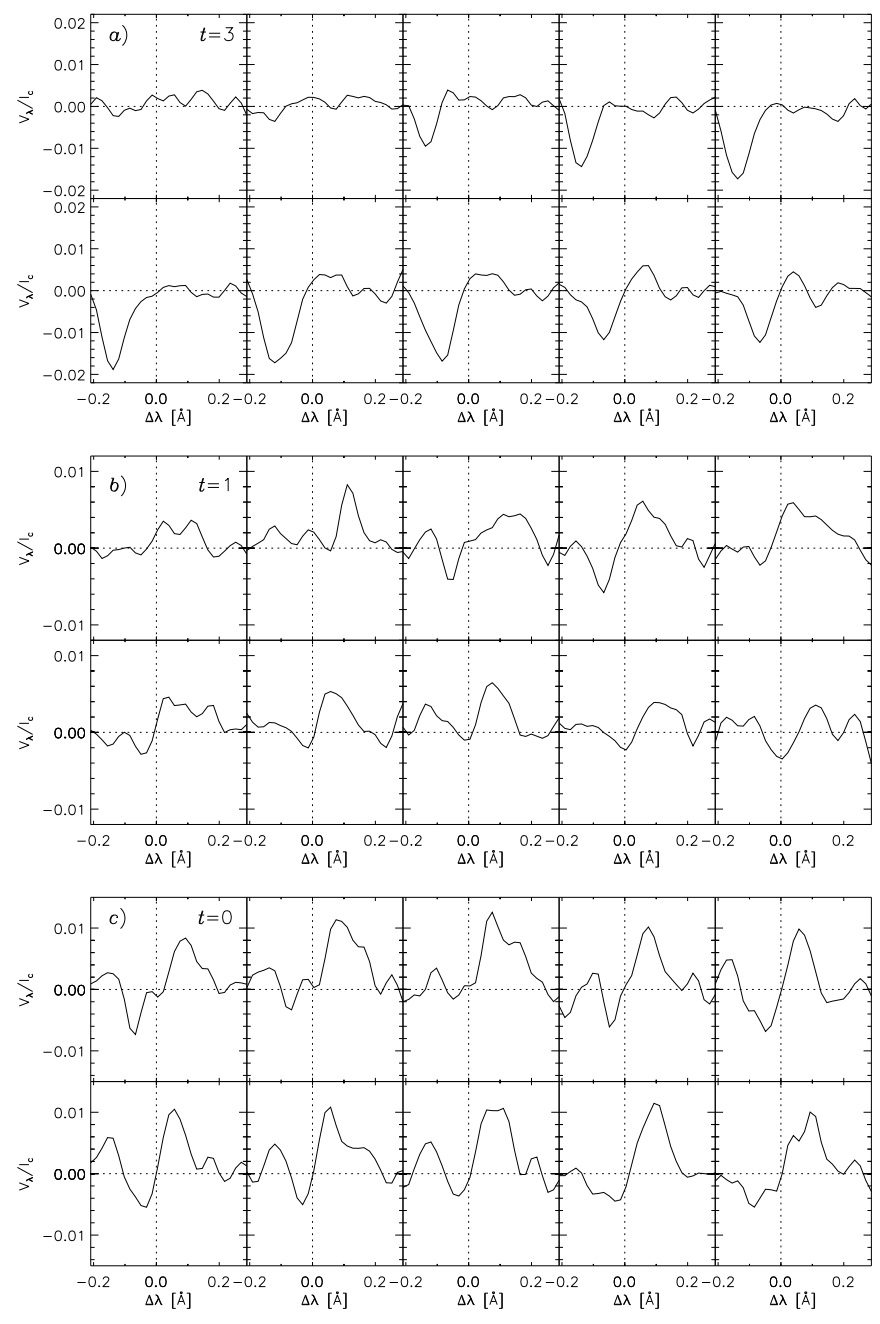

Fig. 8. Temporal evolution of $V$ profiles in IN at the positions a) (upper panel); b) (middle panel); and c) (lower panel) marked in Fig. 7. The numbers given in the panels are the temporal positions, $\Delta t=23 \mathrm{~s}$. Time runs from left to right, first the upper then the lower row. Averages over $0.33 \times 0 ., 33$ are shown.

atmospheres should be subjected to the same caution. We return to this point below in the discussion of the MHD simulations and their comparison with the observations. 

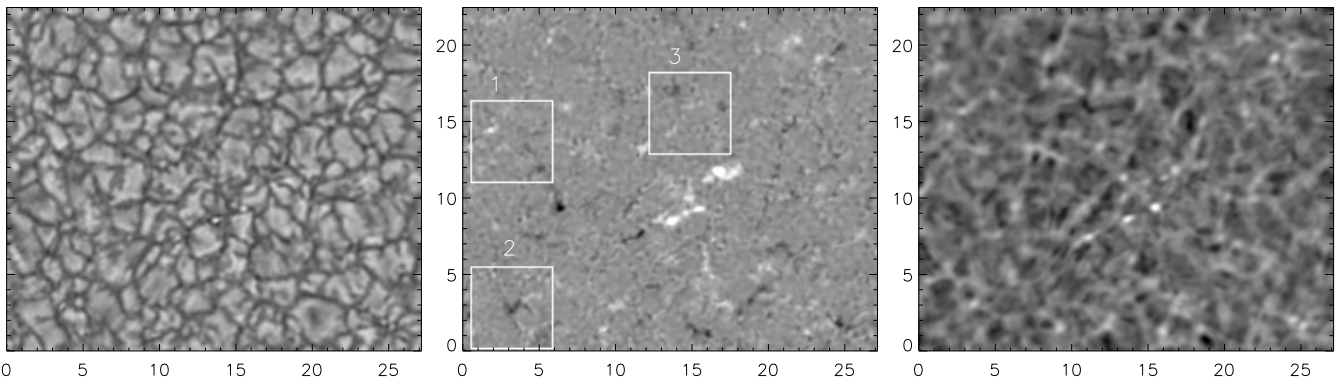

Fig. 9. Overview of the data from July 25, 2007; images from 9.3 after the start of the sequence. From left to right: broadband image, magnetogram clipped at $\left|B_{\mathrm{LOS}}\right|=$ arcsec arcsec $\operatorname{arcsec}$ $100 \mathrm{G}$, line minimum image.
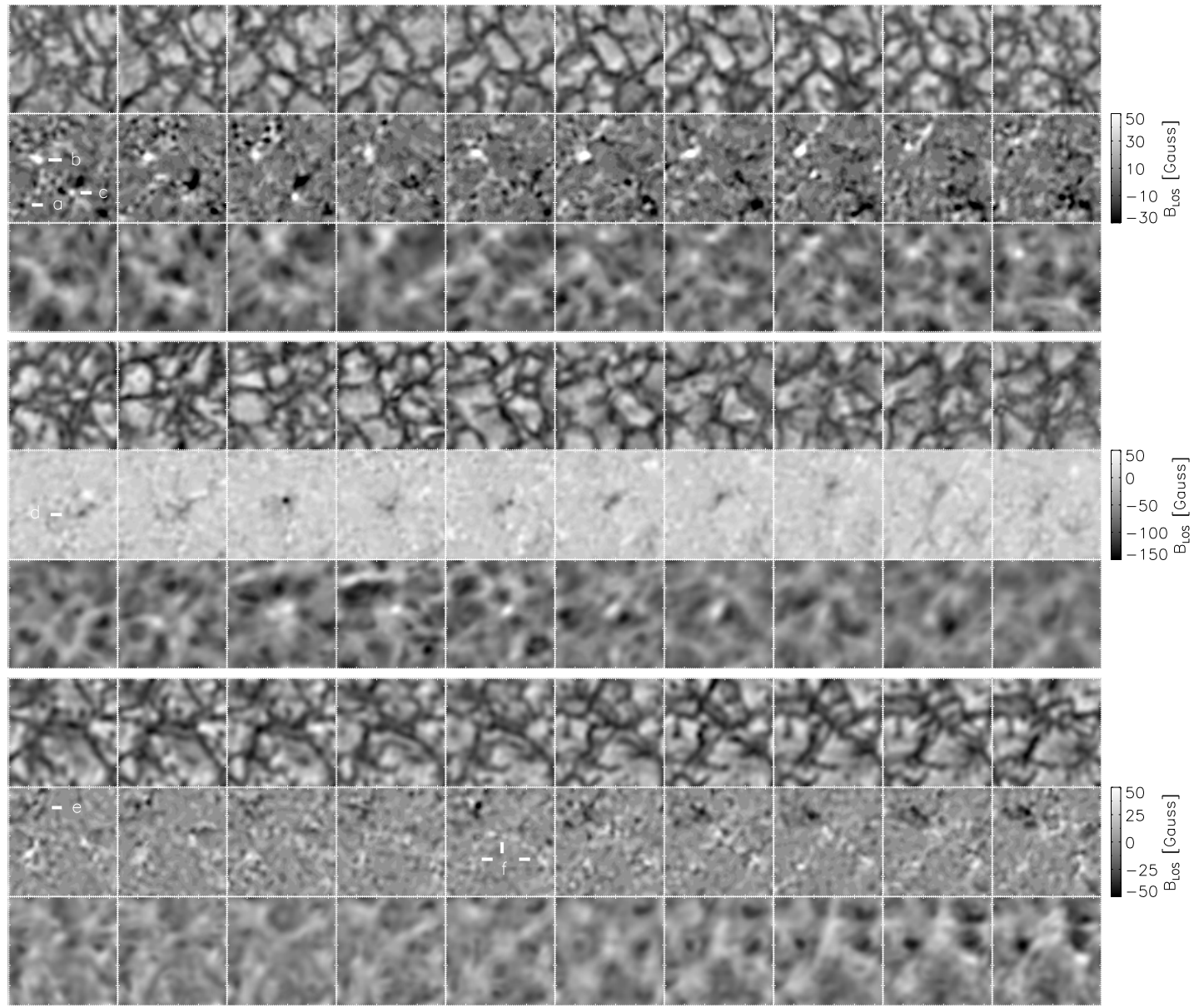

Fig. 10. Three examples of temporal evolution of IN structures from the 2007 July 25 data. For each example, three rows are shown: broadband images, magnetograms, and line minimum images. Only fields with $\left|B_{\mathrm{LOS}}\right|>3.5 \mathrm{G}\left(\sim \sigma_{B}\right)$ are shown, the FOVs are 5'.45 $\times 55^{\prime \prime} 45$. Upper panel: from area "1" in Fig. 9, starting at $t=0 \mathrm{~s}$ of the series, time separation of the columns: $\Delta t=86 \mathrm{~s}$, magnetic fields clipped to $-30 \mathrm{G}<B_{\mathrm{LOS}}<50 \mathrm{G}$; middle panel: from area " 2 ", starting at $t=43 \mathrm{~s}$, time separation of the columns: $\Delta t=129 \mathrm{~s}$, magnetic fields clipped to $-150 \mathrm{G}<B_{\mathrm{LOS}}<50 \mathrm{G}$; lower panel: from area " 3 ", starting at $t=43 \mathrm{~s}$, time separation of the columns: $\Delta t=43 \mathrm{~s}$, magnetic fields clipped to $-50 \mathrm{G} B_{\mathrm{LOS}}<50 \mathrm{G}$.

\subsection{IN evolution, data from July 25,2007}

Figure 9 gives an overview of the second observed quiet area at disc centre at 9.3 after the start of the sequence. We show from left to right: broadband image, magnetogram clipped to $-100 \mathrm{G}<B_{\mathrm{LOS}}<100 \mathrm{G}$, and line minimum image. The magnetograms are somewhat noisier, $\sigma_{B} \approx 3.5 \mathrm{G}$, compared to the magnetograms from the 2007 July 23 data. This was to be expected since the efficiency at measuring Stokes $V$ profiles was lower on July 25, due to measuring the full Stokes vector. The broadband image shows two bright points near the centre of the FOV that also appear bright in the line-minimum image and are related to strong fields, up to $300 \mathrm{G}$ from COG. The movies from the area show that the transient bright points move around and seem to become squeezed into elongated structures. Apart from these, the area appears to be free of network boundary structures. The white rectangles in the magnetogram mark three subareas. The time changes of the structures in these areas will be described in detail below.

When studying the temporal evolution, one obtains the impression that the small-scale magnetic field structures change shape more rapidly than the granular pattern and the line minimum intensity fluctuations. While the granules and the intergranular lanes evolve and move around smoothly in movies with time steps of $\Delta t=43 \mathrm{~s}$, this appears to be a long step for variations in magnetic field structure. To avoid large jumps and trace magnetic fields better along the time, the sequences from July 25, 2007, were smoothed with a subsonic filter with a limiting horizontal velocity of $4.5 \mathrm{~km} \mathrm{~s}^{-1}$. 
We discuss the time variation of the structures in the areas $1-3$ in Fig. 9 by means of the time sequences presented in Fig. 10. There, for each example, the broadband images, the magnetograms, and the line minimum intensities are shown.

1 Upper three rows:

- Magnetic field to the left of the short dash with "a" in the leftmost magnetogram. This field structure is located in the intergranular lane and appears like a chequerboard, or two rows from a chequerboard. Such an appearance is very common among IN fields; the magnetic field changes rapidly there, the pattern is clearly present at the first two snap shots and at later times, but re-organised and displaced; the line minimum intensity above the pattern is marginally enhanced.

- Evolution in the area to the left and above the short dash labelled with " $b$ ". At the beginning of the sequence, a flux concentration within the junction of intergranular lanes $s$ being moved upwards in the magnetograms. It is related with enhanced line-minimum intensity, and in its way, it interacts with other field structures of opposite polarity and seemingly "eats" them. At the same time, towards the upper right of this structure and near the upper border of this sub-FOV, magnetic field inhomogeneities appear together with brightening at line minimum. The two features then move together, producing a further brightening at line minimum.

- Evolution to the left of the dash labelled with "c". Here, a positive polarity field is present close to negative polarity with substantial brightening in line minimum. After few time steps, both fields have dispersed.

2 Middle three rows, negative magnetic polarity to the right of the dash labelled with ' $d$ ' in the first magnetogram:

- This example shows, with time steps $\Delta t=129 \mathrm{~s}$, an intensification of the magnetic field along the time and then a field dilution. The amplitude of the measured field strength here is $-150 \mathrm{G}$ at the time of maximum field strength at time position 3. Inspection of the full granular movie shows proper motion of the granules converging towards the centre of the sub-FOV, i.e. towards the magnetic field structure, during the first phases and, during the final phases, inward motion from two opposite sides and outward flows into the two perpendicular directions. The motion advects fields of both magnetic polarities, and the line-minimum images exhibit intensity enhancement during the phases of maximum magnetic field signal.

3 Lower three rows:

- Magnetic fields with negative polarity to the left of the dash labelled with "e". The temporal evolution of this field structure is fast. One notices advection of magnetic flux with positive and negative polarity. The structure becomes distorted, coagulates with other field strands and splits again; meanwhile, the line minimum intensities are not remarkably bright.

- Intergranular magnetic fields in the half "circle" above the label " $f$ " and indicated with dashes. This example shows especially well the alignment of the fields in intergranular lanes and their development when following in time backward and forward. Again, the evolution of the line minimum intensities does not show especially bright features.

In summary, a description of the ongoing processes is not a trivial task. The dynamics in the visible photospheric and hidden subphotospheric layers are very complex and entangled, and it is not easy to distinguish between cause and effect.

\section{Numerical MHD simulations}

\subsection{MHD simulations}

The simulations were carried out using the compressible 3D radiation MHD code MURaM (Vögler 2003; Vögler et al. 2005). This code solves the MHD equations taking energy exchange via radiative transfer into account as well as the effect of partial ionisation in the equation of state.

The horizontal size of the simulation domain is $6 \times 6 \mathrm{Mm}$, spanned by $288 \times 288$ gridpoints. The height of the domain is $1.68 \mathrm{Mm}$, which is spanned by 120 gridpoints. The resulting gridspacing in the horizontal and vertical directions is 20.83 and $14 \mathrm{~km}$, respectively. Periodic boundary conditions are imposed at the lateral boundaries of the simulation domain. The simulation box extends between $-920 \mathrm{~km}$ below to $750 \mathrm{~km}$ above the average optical depth unity $\left(\tau_{5}=1\right)$ at $500 \mathrm{~nm}$.

The magnetic field above the upper and lower boundaries is constrained to be vertical. The upper and lower boundaries are "open" to plasma motions. The choice of a tall simulation box (750 km above optical depth unity) minimises the influence of the upper boundary conditions on the regions where the considered iron line is formed.

Initially, the simulations begin with a plane-parallel solarmodel atmosphere and no magnetic field. After the purely hydrodynamical convection has reached a statistically steady state, a mixed polarity vertical magnetic field was introduced. The initial configuration of the magnetic field was made such that the simulation domain was split into 4 parts, and the adjacent parts have opposite polarity fields with $200 \mathrm{G}$ amplitude.

The flux is redistributed by convective dynamics, and the opposite polarity fields cancel each other out resulting in an exponential decrease in the overall magnetic flux. Since we are interested in studying the weak internetwork magnetic field, we choose a time sequence of snapshots starting with a mean unsigned field strength of $25 \mathrm{G}$ at $\tau_{5}=1$. The selected time sequence of snapshots covers a time span of $9.2 \mathrm{mn}$ with a cadence of $23 \mathrm{~s}$ between successive snapshots.

To study the observational properties of these simulated small-scale flux concentrations, we calculate the Stokes profiles $I(\lambda), Q(\lambda), U(\lambda), V(\lambda)$ for the iron line Fe I $6173.3 \AA$ with the same atomic parameters as given above in Sect. 2.4. The computations are done in LTE using the STOPRO code in the SPINOR package (Solanki 1987; Frutiger et al. 2000).

The emergent Stokes profiles and the neighbouring continuum intensity are calculated at disc centre for each of the $288 \times 288$ pixels. The thus obtained Stokes profiles are normalised to the local continuum.

We obtained from the emergent Stokes $I$ and $V$ profiles a magnetogram with the COG method, i.e. from the wavelength separation of the COGs of $\frac{1}{2}[I(\lambda)+V(\lambda)]$ and $\frac{1}{2}[I(\lambda)-V(\lambda)]$. Dopplergrams at "high" and "deep" photospheric layers are calculated as well, according to the combinations in Eqs. (2) and (3).

Figure 11 shows the vertical magnetic field component at a height of $150 \mathrm{~km}$ in the left panel, where the maximum of the magnetic field response function is located. The right panels of this figure give vertical cuts extending from $50 \mathrm{~km}$ below average $\tau_{5}=1$ to the top of the simulation box. The vertical magnetic field and the vertical velocities are presented.

Figure 12 depicts some Stokes $V$ profiles. These exhibit exotic shapes, and such profiles are very common. One can generally state that the "strangeness" of the $V$ profiles increases with decreasing amplitudes. Low-amplitude $V$ profiles often possess 

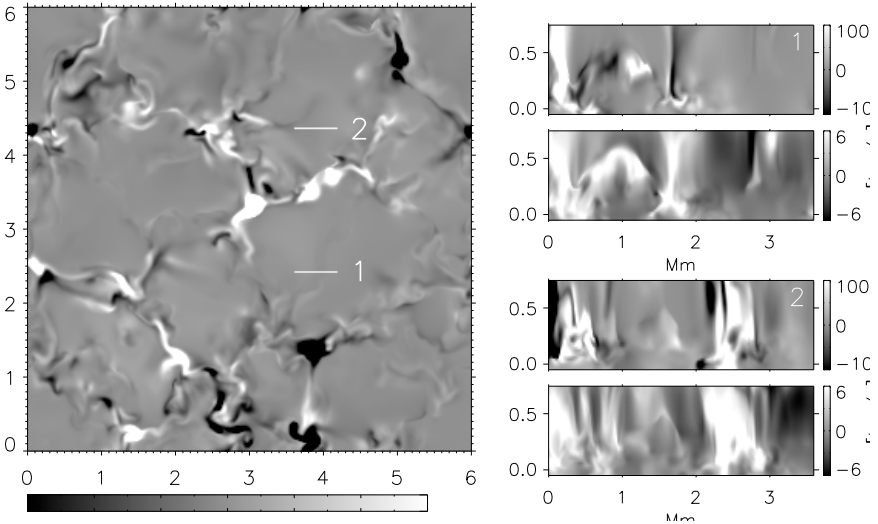

Fig. 11. Left: vertical magnetic field component at $150 \mathrm{~km}$ with bright indicating direction outward of the atmosphere and dark direction inward. Right: sections of vertical cuts through the atmosphere to the left of the lines indicated by " 1 " and " 2 " in the left panel; upper panels: vertical magnetic field components with bright indicating direction outward and dark inward; lower panels: vertical velocities with bright upward and dark inward motion. The sections extend from from $-50 \mathrm{~km}$ to $+750 \mathrm{~km}$, with average $\tau_{5}=1$ at $0 \mathrm{~km}$. The magnetic field presentations are limited to $\left|B_{\text {vert }}\right|<100 \mathrm{G}$, the velocities to $\left|v_{\text {vert }}\right|<6 \mathrm{~km} \mathrm{~s}^{-1}$.

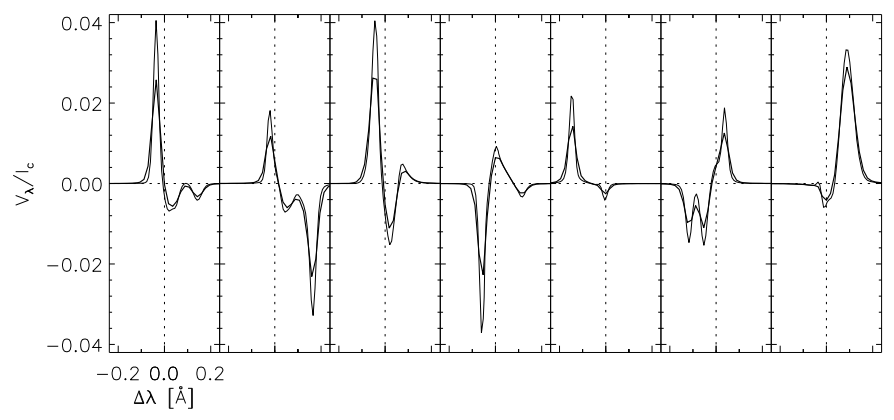

Fig. 12. Stokes $V$ profiles from MHD simulations; thin: original profiles, thick: after degradation in wavelength (see Sect. 4.2).

four to five lobes. The structures with the largest magnetic field in the simulation box have two-lobed $V$ profiles with amplitudes $\sim 0.3 \times I_{\mathrm{c}}$, which are often very asymmetric. We also note without showing that the zero crossings of the strong $V$ signals exhibit rapid changes across the magnetic features fluctuations of velocities of $\left|v_{\mathrm{LOS}}\right| \approx 4 \mathrm{~km} \mathrm{~s}^{-1}$. On the one hand, $V$ profiles with large amplitudes, thus rather simple shapes, can be subjected to inversion calculations from which trustworthy, approximate atmospheric parameters can be retrieved. On the other hand, $V$ profiles exhibiting complex shapes like those observed (e.g. Fig. 4, two profiles on the right, and Fig. 8) and as those from MHD simulations would be difficult to interpret by inversions assuming a few atmospheric parameters, e.g. by a MilneEddington inversion. In view of the magnetic field structure in Fig. 11 and of the profiles in Fig. 12, the hypothesis of MIcroStructured Atmosphere (MISMA, Domínguez Cerdeña et al. 2006, and references therein) appears a viable concept for retrieving the magnetic and velocity structure, yet with sufficiently resolved observations.

\subsection{Degradation to resolution of observations}

For comparison with the observations, the observable intensities and Stokes profiles that emerge from the simulation box had to be degraded to the resolution of the observations in both

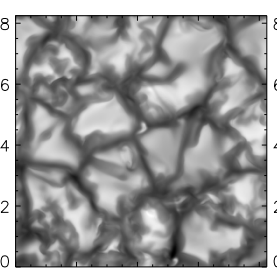

$$
\text { arcse }
$$

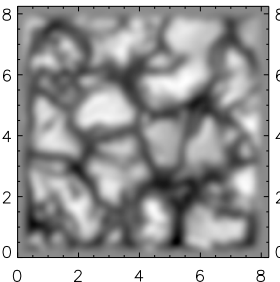

$\stackrel{4}{\operatorname{arcsec}}$

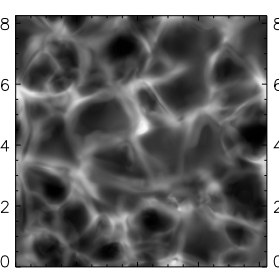

4
$\operatorname{arcsec}$

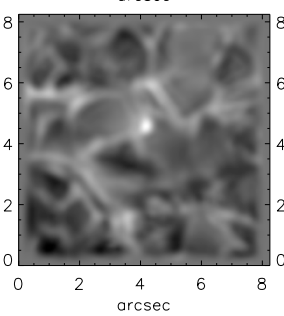

Fig. 13. Effects of degradation. Upper row from original simulation data, lower row after full degradation to quality of observations, from left to right: intensity in continuum near $\mathrm{Fe} \mathrm{I}$ line, intensity at local line minimum, $B_{\mathrm{LOS}}$ from COG determination with the field strength limited to $\left|B_{\mathrm{LOS}}\right| \leq 50 \mathrm{G}$. We adopt $1^{\prime \prime} \widehat{\equiv} 725 \mathrm{~km}$ on the Sun. The degraded maps are apodised at their borders from filtering.

wavelength and spatial resolution. The degradation consisted in several steps. The first degradation occurs intrinsically, by the transfer of the Stokes vector through the photosphere. This is seen when comparing the spatial structure of the vertical magnetic field at a height of $150 \mathrm{~km}$ in Fig. 11 with the magnetogram in the upper right corner of Fig. 13. The small-scale alternation of magnetic polarity has mostly disappeared because many different atmospheric layers contribute with variable polarity fields and with their vertical velocities of opposite flow direction (cf. vertical cuts in Fig. 11). The Stokes $V$ profiles from various positions in the (simulated) FOV in Fig. 12 are correspondingly very abnormal.

Next we scaled the wavelength resolution and step width to the spectrometer's resolution and step width. Obviously, the COG magnetic field measurement is very robust against spectral degradation since the COG method itself is an averaging method. Broad $V$ profiles with large amplitude from strong field structures are little affected by the limitation in spectral resolution. Some degraded $V$ profiles are shown with the original profiles in Fig. 12. The wavelength degradation preserves the shape of the profiles well, but decreases them in amplitude.

The main effect comes from the limited spatial resolution. To account for the latter we convolved the simulated data in spatial direction with a normalised point spread function that contains the telescopic Airy function and a 5\% contribution from

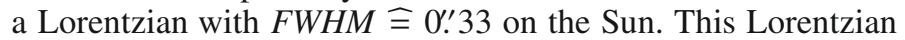
with its extended wings is chosen to account for scattered light by telescope and Earth's atmosphere from regions with few arcsec distance. Additionally a "wide-angle" scattering has been applied with an amplitude of $0.15 \bar{I}_{\lambda}$, where $\bar{I}_{\lambda}$ is the intensity at this specific wavelength $\lambda$ averaged over the FOV. Finally, the images were filtered with the spatial low-pass filter to scales $\geq 0$ '. 33 (dashed filter in Fig. 1). The image resolution of $20.83 \mathrm{~km}$ was then binned to the pixel size of the detectors in the observations corresponding to $79 \mathrm{~km}$. The spatial degradation brings the simulated rms continuum fluctuations from $0.17 I_{\mathrm{c}}$ to the observed fluctuations of $0.08-0.09 I_{\mathrm{c}}$. At the same time, the average unsigned LOS component decreases from $16-17 \mathrm{G}$ at $150 \mathrm{~km}$ to 5.1-5.2 G from COG measurement. 


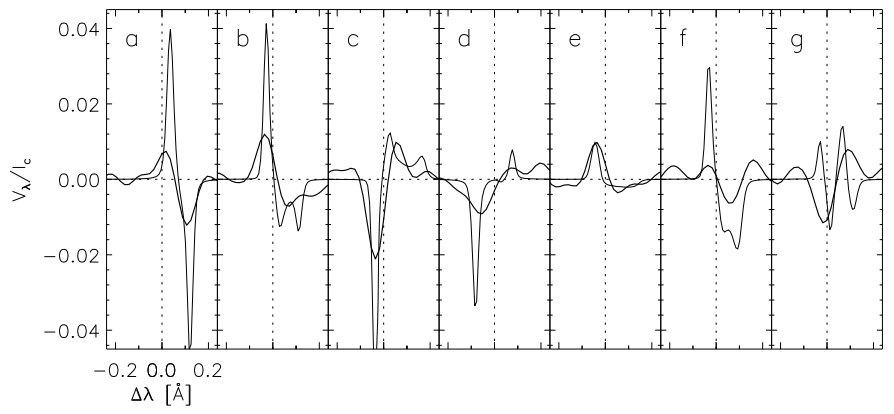

Fig. 14. Comparison of $V$ profiles from original MHD simulations (thin) and from the degraded data set thick, latter multiplied with a factor of 2 . The profiles stem from the eleventh temporal position of the simulated time sequence.

The last degradation deals with the noise. We added Gaussian noise proportional to the intensities in the $(I+V)$ and $(I-V)$ profiles and filtered again in space and wavelength with the filters depicted in Fig. 1. The noise amplitude was chosen to obtain noise in the magnetic field measurements of $\sim 2 \mathrm{G}$.

Some of the effects are shown in Fig. 13 for the degradation of the intensity images and magnetograms. This figure stems from the first temporal position of the simulations with calculated profiles. The bright small-scale structure at the centre of the FOV of the continuum images has a maximum intensity of $1.4 \times \bar{I}_{\mathrm{c}}$, so not higher than the brightest granular intensities. Its intensity in the image degraded to the resolution of the observed, speckle reconstructed broadband images (0.25) is only $\Delta I=0.05 \times \bar{I}_{\mathrm{c}}$ above the dark background, so it cannot be considered as a typical bright point.

Figure 14, from the 11th temporal position in the simulations, shows examples of $V$ profiles from simulations (thin curves) and from their full degradation (thick). The amplitudes of the degraded profiles are multiplied with a factor of 2 , for the presentation. We comment without showing that the largeamplitude, original profiles from the strong fields are generally reproduced well, apart from some broadening. The main reason for this broadening is the rapid fluctuation of field strength and velocity across the magnetic structure. Noise filtering has a minor effect. Similarly for low-amplitude $V$ profiles, a slow variation of the atmospheric structure including magnetic fields yields slowly varying $V$ profiles across the resolution element of the degraded data.

The $V$ profile "a" in Fig. 14 is redshifted and two-lobed. The degraded profile almost has a factor of 10 smaller amplitude than the original one and is less redshifted. The degraded profiles " $b$ " and "c" allow the strong asymmetry to be seen. Profile "d" has only a tiny red lobe that is buried in the noise after degradation. The original $V$ profile "e" is reproduced well in the degraded profile, apart from the amplitude and the broadening. These last two profiles " $d$ " and " $e$ " stem from regions with variation that it is not too fast across the resolution element. In contrast to them, the complex profiles " $f$ " and "g" are from areas with rapid variation. Thus the degraded profiles, albeit not consisting of pure noise, exhibit no resemblance with the original ones but are apparently a mixture of very different profiles.

Test calculations have shown that one needs low-noise data with a spatial resolution of 0 . $^{\prime} 1$ or better to preserve the Stokes profiles, at least the shapes of the $V$ profiles, while it will be extremely difficult to preserve their amplitudes.

In Table 2 we summarise the effects of the various degrees of degradation in different parameters, i.e. the line-of-sight (LOS) components of unsigned magnetic field $\left\langle\left|B_{\mathrm{LOS}}\right|\right\rangle$ averaged over field of view, minimum and maximum of $B_{\mathrm{LOS}}(\min \max )$, area coverage of fields $|B| \geq 3 \sigma_{B}$ (Cov), and rms values of LOS velocities at deep and high layers. Index lim in the third column indicates limitation to $\left|B_{\mathrm{LOS}}\right| \leq 700 \mathrm{G}$ in the original COG "measurements" and elimination of those pixels with larger field strengths. The first two rows in the table refer to the simulation box, and the other rows to Stokes profiles.

One notices a decrease in $\left|B_{\mathrm{LOS}}\right|$ by a factor 4-5, from the intrinsic field to the detected field. The effect of limited spatial resolution is the strongest. We have given two values for the average unsigned magnetic field strengths on the following grounds. The area contains some parts with kilo-G fields. Although their number of pixels in the FOV is small, a few tens, as seen from the histograms in Fig. 17, they contribute to the average unsigned field. To eliminate their effect, we chose to include in the averaging only areas with \langle|$B_{\mathrm{LOS}}$,im |\rangle smaller than $700 \mathrm{G}$ in the $\mathrm{COG}$ measurements of the non-degraded data. Also, since the observations contain noise of $\sigma_{B} \approx 2 \mathrm{G}$, the fields of those pixels with $\left|B_{\mathrm{LOS}}\right|<3 \sigma_{B}$ were set to zero in the averaging, in both the simulated and the observed data sets.

The entries for the observations from this work stem from an IN area with little to no strong magnetic flux. The average LOS magnetic field strengths of $3 \mathrm{G}$ is from the observing day 2007 June 23. The initial conditions for numerical MHD simulations were actually chosen to obtain $\left\langle\left|B_{\mathrm{LOS}}\right|\right\rangle \approx 3 \mathrm{G}$ after the full degradation process.

The velocities are included in Table 2 to show the overshooting granular flows. The 5-min oscillations do not contribute since they are removed from the observed data by subtraction of velocities smoothed in spatial co-ordinates. The velocity amplitudes determined from the formulae in Eqs. (2) and (3) can be high, in deep layers up to $\left|v_{\mathrm{LOS}}\right|=4.5 \mathrm{~km} \mathrm{~s}^{-1}$, and in high layers in the range of $-3 \leq v_{\mathrm{LOS}} v_{\mathrm{LOS}} \leq 2 \mathrm{~km} \mathrm{~s}^{-1}$. The degradation in wavelength decreases the rms velocity at deep layers and increases it at high layers due to increased mixing of contributions from different heights of formation.

\subsection{Temporal evolution in simulations}

The degraded data sets, including the $V$ profiles at each wavelength position, were subjected to a subsonic filter with a cutoff of $7.5 \mathrm{~km} \mathrm{~s}^{-1}$, as the observations. Figure 15 gives three examples of the temporal evolution of the (degraded) numerically simulated continuum intensities, line minimum intensities, and COG magnetograms in Fe I $6173 \AA^{1}$. Time runs from top to bottom, and the initial times are given in the upper left corners of the continuum images. The second set of columns are continuations of the first set.

The coarse distribution of the strong magnetic fields stays the same during the full sequence, and yet the finestructures, especially those of the weaker fields, change from one temporal position to the next. This is seen in the accompanying movie (see footnote). It illustrates the need for high temporal resolution. A cadence of $46 \mathrm{~s}$ gives some jitter of the small-scale fields from one time step to the next, which was seen also in the observations.

Generally, the magnetic fields detectable in the magnetograms from the degraded MHD simulations are located in the intergranular structures seen in the continuum images. The

\footnotetext{
1 The full time sequence, containing the original and degraded simulated continuum intensities, line minimum intensities, magnetograms, and Dopplergrams, is available as a gif movie as online material.
} 
Table 2. Values of various parameters from observations and from simulations at different states of degradation.

\begin{tabular}{lcccccc}
\hline \hline Method & $\left\langle\left|B_{\mathrm{LOS}}\right|\right\rangle[\mathrm{G}]$ & $\left\langle\left|B_{\mathrm{LOS}, \mathrm{lim}}\right|\right\rangle[\mathrm{G}]$ & $\min \max [\mathrm{G}]$ & $\operatorname{Cov}$ & $v_{\text {deep }, \mathrm{rms}}\left[\mathrm{km} \mathrm{s}^{-1}\right]$ & $v_{\text {high,rms }}\left[\mathrm{km} \mathrm{s}^{-1}\right]$ \\
\hline$B$ at $150 \mathrm{~km}$ & 17.4 & 16.1 & $-1520 / 1710$ & & 1.8 & 1.0 \\
$v_{\text {rms }}$ at $100 \&$ \& $300 \mathrm{~km}$ & & & & & 1.7 & 1.0 \\
COG, original & 12.1 & 10.3 & $-1170 / 1470$ & 1.5 & 1.1 \\
COG, degr $\lambda$ & 12.0 & 10.3 & $-1170 / 1470$ & 0.92 & 0.58 \\
COG, degr $x y$ & 5.4 & 5.1 & $-210 / 270$ & & 0.76 & 0.68 \\
COG, degr $x y, \lambda$ & 5.5 & 5.1 & $-200 / 250$ & & 0.65 & 0.66 \\
COG, degr $x y, \lambda+$ noise & 3.6 & 3.2 & $-180 / 270$ & 0.17 & 0.59 & 0.47 \\
COG, observ, this work & & 3.0 & $-47 / 33$ & 0.30 & & \\
\hline
\end{tabular}
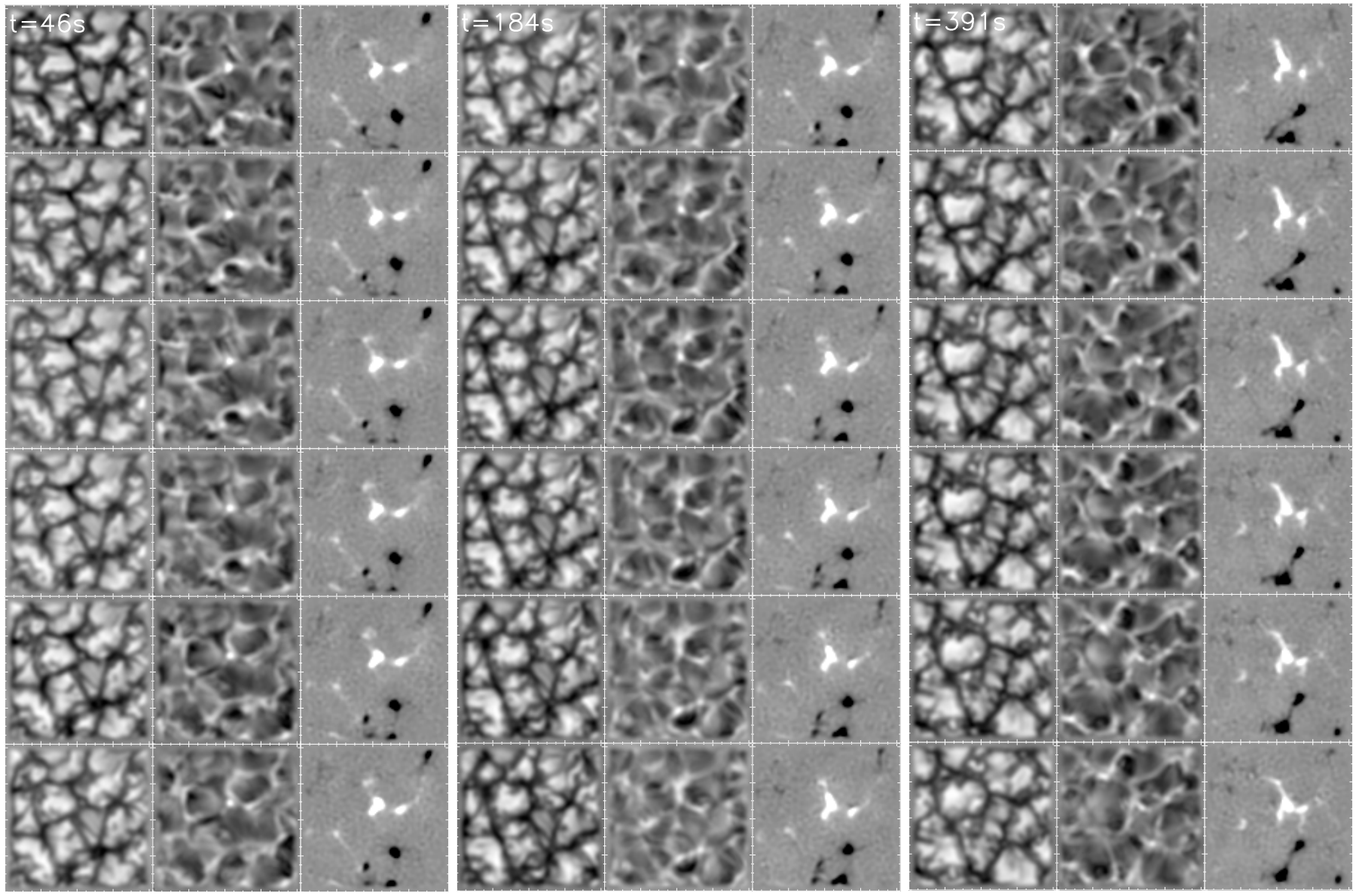

Fig. 15. Temporal evolution in numerically simulated data set. Three sequences from the fully degraded data are shown, the first two from the first half of the series, the third from its end. The (sub)columns show from left to right: continuum intensity, line minimum intensity, and $B_{\mathrm{LOS}}$ clipped to amplitudes $\leq 50 \mathrm{G}$. Time runs from top to bottom at a cadence of $23 \mathrm{~s}$ with the initial times given in the top left panels. FOV is 8 '. $28 \times 88^{\prime \prime} 28$ (1" $\widehat{=} 725 \mathrm{~km}$ on the Sun).

original data also show weak fields in the granules (cf. Fig. 13). Some apparently "diffuse" fields, such as in the upper left corner at the initial time of the sequence, are related to small convection elements of sub-arcsec size.

The strong positive polarity field close to the centre of the FOV has its counterpart as brightening in the line minimum images. We mentioned above that it does not have the properties of a bright point discussed in the observational part, together with Fig. 5. It is more like a line gap feature with reduced line depression. In fact, within this structure, the Stokes I profiles exhibit clear Zeeman splitting.

What is missing in the magnetograms are the many structures in the intergranular lanes with opposite magnetic polarity next to each other. They were seen in the observations (cf. Figs. 7 and 10) with field strengths from COG of 10-50 G, well above the noise. More recent numerical MHD simulations with the MURaM code, using a very high spatial resolution and correspondingly high magnetic Reynold's number, exhibits dynamo action and has many more of these features with close opposite polarity (Schüssler \& Vögler 2008; Danilovic et al. 2008).

Figure 16 depicts two examples of $V$ profile evolution. Here also the degraded profiles were multiplied by a factor of 2 . The examples are selected outside the strong flux regions in the FOV. In the upper sequence of this figure, the $V$ profiles develop, starting with two lobes and rapidly varying amplitude and asymmetry. During the first nine time positions, the degraded profiles 

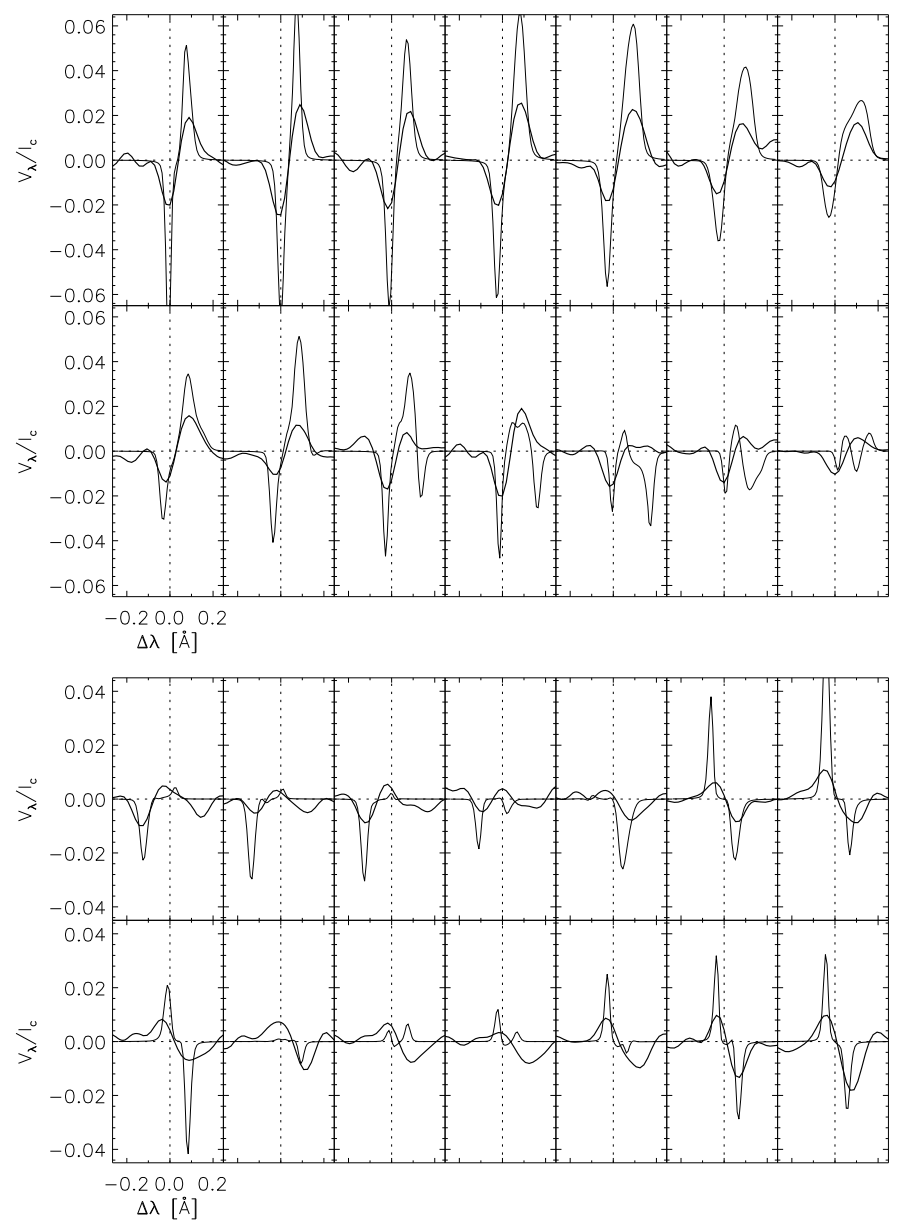

Fig. 16. Two examples of temporal evolution of $V$ profiles from MHD simulations, with $23 \mathrm{~s}$ cadence and at fixed positions in the FOV. Time runs from left to right, first upper rows then lower rows. Thin profiles: from original data; thick profiles: from fully degraded data, multiplied with a factor of 2 and averaged over $0.33 \times 0 . \prime 33$.

follow the original ones closely, apart from strong amplitude reduction of the former. The positions of the zero crossings, which indicate downflows with up to $1.5 \mathrm{~km} \mathrm{~s}^{-1}$, are reproduced well. Nevertheless, towards the end of the sequence, starting from time position 10 (second row, third panel), the original profiles weaken and become very abnormal. The degraded profiles cannot follow this evolution. They represent some averages over the resolution element plus noise.

The second example in the lower part of Fig. 16 shows rapid development of the original one-lobed $V$ profile and then a reversal of magnetic polarity within $23 \mathrm{~s}$, between time positions 4 and 5. At these positions, the negative $V$ lobe changes from being on the blue side to the red side of the zero wavelength position. The degraded profiles can reproduce the polarity change, although with noise and much broader than the original profiles. In view of the abnormal, nearly single-lobed $V$ profiles, it is better to state that one sees a change in dominating polarity along the LOS. During the second half of the sequence (lower row), the $V$ profiles weaken and increase again, reappearing first with a one-lobed profile, then with two lobes. As in the first example above, the degraded $V$ profiles do not reproduce the very abnormal, low-amplitude original profiles, but find the correct polarity, at least for original profiles with sufficient amplitude.
We note from inspection of the movies that the temporal changes in the profiles result from both local evolution and lateral convective flux transport, as expected.

\section{Discussion}

In this section we first compare the observational results with those from the numerical MHD simulations. Then we compare our results on IN fields with other work.

\subsection{Comparison of observations and simulations}

A comparison of the observations with the numerical simulations has two aspects. First, one can discuss general similarities and dissimilarities. Second, one has to degrade the simulation outcome to the quality of the observations. This allows to see the processes that are behind the observations, but hidden by the lack of resolution. Thus the simulations are extremely valuable for the interpretation.

Without attempting completeness, we list several points in this comparison:

1. As known from earlier studies of MHD processes in the granulation, the magnetic fields are mainly located in the intergranular lanes as observed. They are distributed by the convection processes. The reaction of the magnetic fields is fast. Therefore, it turns out that time steps of $\sim 40 \mathrm{~s}$ and longer are too long for studying processes on scales of 0.3 and smaller.

2. The simulation results show that the MHD processes are much more complex than observations suggest, even at the best spatial resolution. The often very abnormal $V$ profiles result from rapid changes in magnetic fields, in their polarity, and in velocities of several $\mathrm{km} \mathrm{s}^{-1}$ along the LOS.

3. The non-degraded, as well as the degraded, simulated "measurements" of the magnetic fields do not exhibit chains of positive and negative polarity fields next to each other in intergranular lanes. Possibly a much higher magnetic Reynolds number is needed for a more adequate concept for IN fields showing more alternating polarities (Schüssler \& Vögler 2008; Danilovic et al. 2008).

4. Related to this last point is that, after we had degraded the simulation output to the best of our knowledge, the interganular lanes show less field coverage in the simulations, $17 \%$ according to Table 2 , than in the observations ( $30 \%$ coverage).

5. Some of the areas in the simulation surface appear more like network with kilo-G fields even after degradation as described above, although the transition from network to internetwork fields is not sharp. In the observational results, we have excluded such areas from the study of IN fields. Possibly, this is again an outcome of the simulation initialisation, and a numerical simulation with a surface dynamo action starting from a low-amplitude seed field could yield less network features.

6. In the observations, bright points were found with high broadband intensities well above the brightest granular level. They represent transient processes with fast downflows on small scales. We did not find any such features in the simulation - possibly because the bright points are relatively rare and the simulation box was small ( $6 \mathrm{Mm}$ by $6 \mathrm{Mm}$ in the horizontal directions) and of limited duration $(9.2 \mathrm{mn})$. A longer time series or a bigger box are obvious targets for future simulations. 


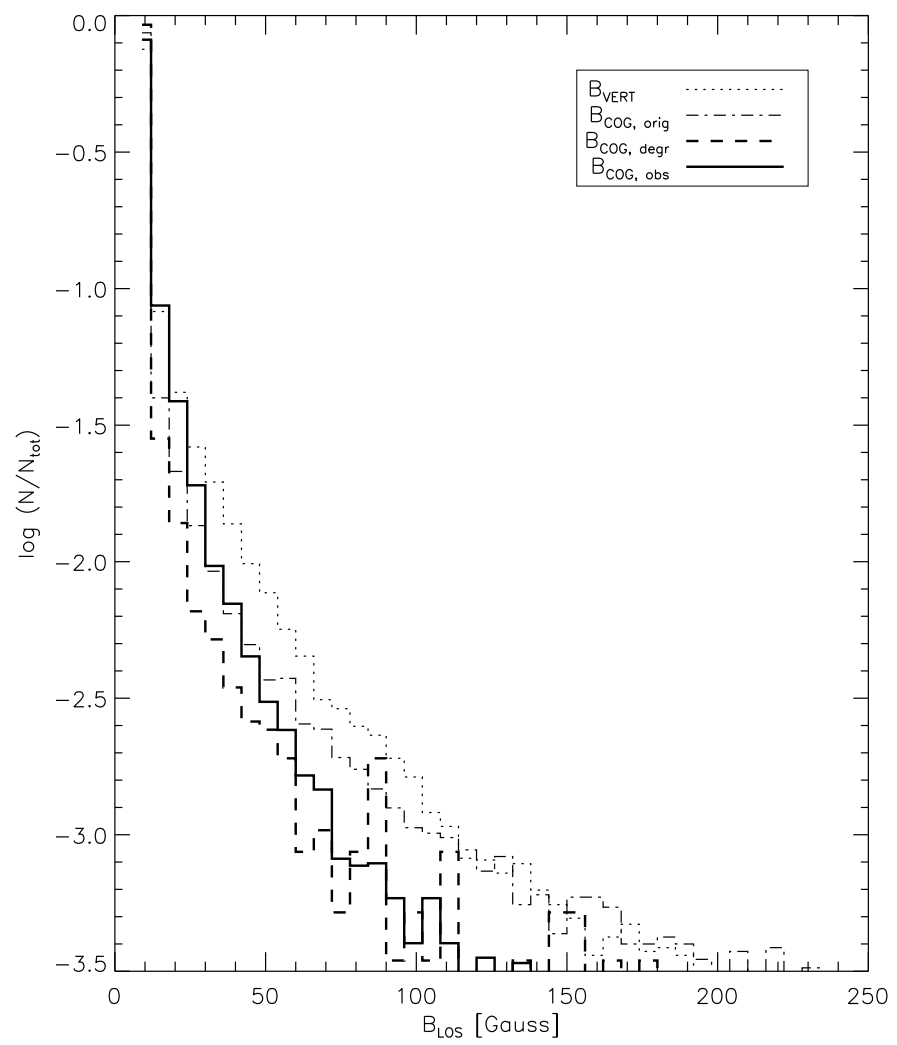

Fig. 17. Histograms of $\left|B_{\mathrm{COG}}\right|$ from observations and numerical simulations determined with the COG method, except for $\left|B_{\text {vert }}\right|$ which is from the simulation cube at $150 \mathrm{~km}$ above $\tau_{5}=1$.

A further comparison is made in Fig. 17. There the distributions of $\left|B_{\mathrm{COG}}\right|$ are depicted. The simulation data stem from the first time postion of the simulation with computed Stokes profiles. Here, $\left|B_{\text {vert }}\right|$ denotes the vertical (LOS) magnetic field component at $150 \mathrm{~km}$ above $\tau_{5}=1$. The data for the observations were from the whole FOV in Fig. 3. For a proper comparison, the pixels with low fields, $\left|B_{\mathrm{COG}}\right| \leq 6 \mathrm{G}$, were removed from the counts and the remaining numbers were then normalised by the total remaining pixel numbers in the FOVs.

Certainly, fields exist with strengths above $250 \mathrm{G}$, but very few compared to those that entered the histograms of Fig. 17. The interesting part here is the one with low apparent field strengths, below $250 \mathrm{G}$. Both, $\left|B_{\text {vert }}\right|$ and $\left|B_{\mathrm{COG}, o r i g}\right|$ exhibit very similar distributions at fields larger than $100 \mathrm{G}$. These fields and COG "measurements" are from areas with one polarity strongly dominating any mixed polarity situation along the LOS. Below $\sim 20 \mathrm{G}$, both distributions are also similar. These data are from granules, with many pixels exhibiting very weak fields. Between $20 \mathrm{G}$ and $100 \mathrm{G}$, the original "measurements" show less fields than at $150 \mathrm{~km}$, and they are intrinsically degraded by radiative transfer where mixed polarities are playing a role.

An important result in Fig. 17 is the difference between the histograms of the observed COG fields and the fully degraded COG fields. In the range of $\sim 10 \ldots 100 \mathrm{G}$, the latter histogram is lower than the one from the observations by a factor 2 to 3 . This explains the lower area coverage of the degraded fields compared to the observed fields in Table 2 and also the lower appearance of fields in the $\sim 10 \ldots 100 \mathrm{G}$ range in Figs. 13 and 15 from the simulations compared with Figs. 7 and 10 from the observations.
Table 3. Line-of-sight components of unsigned IN magnetic field $\left\langle\left|B_{\mathrm{LOS}}\right|\right\rangle$, averaged over field of view, at disc centre.

\begin{tabular}{|c|c|c|}
\hline$\overline{\text { Source }}$ & Method & 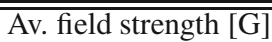 \\
\hline Khomenko et al. (2003) & IR lines & $2.7-4$ \\
\hline Domínguez Cerdeña & speckle \& & \\
\hline Lites \& & line ratio & $17-21$ \\
\hline $\begin{array}{l}\text { Socas-Navarro (2004) } \\
\text { Orozco Suárez }\end{array}$ & $V_{\text {tot }}$ & $8-10$ \\
\hline et al. (2007) & inversion & 3.4 \\
\hline Lites et al. (2008) & $V_{\text {tot }}$ & 6 \\
\hline $\begin{array}{l}\text { Martínez González } \\
\text { et al. (2008) }\end{array}$ & inversion & 4.1 \\
\hline Bello González & speckle \& & \\
\hline \& Kneer (2008) & COG & 2.2 \\
\hline $\begin{array}{l}\text { Trujillo Bueno } \\
\text { et al. (2004) } \\
\text { this work: }\end{array}$ & Hanle & $\sim 100$ \\
\hline observed & $\begin{array}{l}\text { speckle \& } \\
\text { COG }\end{array}$ & $3.0-4.0$ \\
\hline simulated, original & at $150 \mathrm{~km}$ & 16.1 \\
\hline simulated, original & $\mathrm{COG}$ & 10.3 \\
\hline simulated, degraded & $\mathrm{COG}$ & 3.2 \\
\hline
\end{tabular}

\subsection{Comparison with other work on IN fields}

In Table 3, we collect values from the literature and from this work for the unsigned LOS component of the internetwork magnetic field averaged over the FOV.

Khomenko et al. (2003) have used full Stokes measurements in the $\mathrm{Fe}$ I lines at $1.56 \mu$ to determine the field strengths $B$, inclination angles $\gamma$ and filling factors $f$ (in their notation). With their $f \leq 0.02$ in approximately $50 \%$ of the FOV and $B=250-300 \mathrm{G}$, one obtains $\langle B\rangle \approx 0.5 \times 0.02 \times 270 \mathrm{G}=2.7 \mathrm{G}$. The authors themselves give $\langle B\rangle=f \cos \gamma B=8 \mathrm{G}$ averaged over approximately $50 \%$ of the IN area with a significant signal. Domínguez Cerdeña et al. (2003) performed speckle reconstruction of polarimetric images in the Fe $16302 \AA$ line pair and conclude with many strong fields from the line ratio. With ASP measurements in the same line pair, Lites \& Socas-Navarro (2004) performed a normalised integration over the Stokes $V$ profile, $V_{\text {tot }}=\int\left|V_{\lambda}\right| \mathrm{d} \lambda / \int I_{\mathrm{c}} \mathrm{d} \lambda$ and calibrated this quantity. The value given by Orozco Suárez et al. (2007) is from inversion of SOT-HINODE measurements, again in the $6302 \AA$ line pair. IN measurements from other SOT-HINODE data and the calibration with fields in strong magnetic flux regions lead Lites et al. (2008) to an unsigned average $\left|B_{\mathrm{LOS}}\right|=6 \mathrm{G}$. Martínez González et al. (2008) simultaneously measured the full Stokes parameters in the IR and the $6302 \AA$ line pairs and also carried out inversions simultaneously in all four lines. In the work of Bello González \& Kneer (2008), the COG method was also applied to speckle reconstructed data to measure the LOS components of the magnetic field. From the fields with $\left|B_{\mathrm{LOS}}\right|>3 \sigma_{B}$, the data from July 23,2007 , give an average IN field $\left|B_{\mathrm{LOS}}\right|$ of $3 \mathrm{G}$, those from July 25, 2007, give 4.0 G. By means of the Hanle effect, Trujillo Bueno et al. (2004) found unresolved, mixed polarity ("hidden") fields, which are possibly entangled on micro-scales.

The average unsigned LOS field component found in the present investigation from observation compares satisfactorily with values found by Khomenko et al. (2003), Orozco Suárez et al. (2007), the value in Lites et al. (2008), and the one in Martínez González et al. (2008). As noted by Lites \& Socas-Navarro (2004), the field strength obtained in our observations and also in the Orozco Suárez et al. (2007) measurements 
is not larger than from other data despite the high spatial spatial resolution and the high sensitivity.

The entries in the lines "simulated" in Table 3 refer to the MHD simulations. The values of $\left|B_{\mathrm{LOS}, \mathrm{lim}}\right|$ from Table 2 are repeated here. After degradation in spectral and spatial resolution and after adding an appropriate amount of noise, the simulations were designed to give approximately $3 \mathrm{G}$ for the average LOS field component measured with COG. At an atmospheric height of $150 \mathrm{~km}$, from where we receive the magnetic signal with the COG method (cf. Fig. 2b), we obtain $\left\langle\left|B_{\text {Los }}\right|\right\rangle \approx 16$ G. After degradation in wavelength and spatial resolution, this value has decreased by a factor of 3 . Then, by adding noise and analysing only the $V$ profiles with amplitude $3 \sigma_{B}$ above noise, the average unsigned field strength has again decreased by a factor of 1.7 .

\section{Conclusions}

The observations presented in the first part of this study revealed highly dynamic processes in the solar photosphere. The fast observational cadences allowed study of important new phenomena and dynamics. Some examples are given in this study, and more can be found in Bello González et al. (2008). The efforts to combine $2 \mathrm{D}$ spectropolarimetry of high sensitivity with efficient data gathering and image reconstruction were successful. At present, the results from the observations and data analysis are among the best to our knowledge. The data will be subjected to further analyses for more in-depth studies of the smallscale magnetic field dynamics of the quiet solar photosphere. We demonstrated that especially the investigation of temporal evolution in photospheric magnetoconvection has become possible.

Likewise, the numerical MHD simulations used here contain many more details than could be presented in this study. They contain a wealth of information on MHD processes in a stratified hot plasma to be uncovered in future investigations.

The combination of high spatial resolution observations and MHD simulations turned out to be very fruitful. We have learned much from the comparison of observational results and simulations. Much we have seen in the brightness, magnetic field, and velocity evolutions is also reflected in the simulations. And not all observational results are reproduced by the simulations, such as bright points and changes in polarity along inter-granular lanes. Possible reasons for these discrepancies include the relatively low magnetic Reynolds number in the simulations and the smallness of the simulation box that leads to limited statistics.

Most importantly, the simulations have taught that the actual properties of the magnetic photosphere is much more complex at small scales than the observations, although of high quality, suggest. The many abnormal Stokes $V$ profiles turn out to be the normal case in simulations. The observation of such profiles is best performed by comparing with the simulations and the physical conditions producing such profiles.

In view of the results from the numerical MHD simulations and in comparison with the observations, there is still a long way to go for observational improvement. The spectral resolution of the "Göttingen" FPI spectropolarimeter appears to be adequate. As the degradation of the simulated $V$ profiles have shown, the spectrometer by itself can even reproduce the shape of very abnormal $V$ profiles with four to five lobes, although with reduced amplitude. Sacrificing the spectral purity for the sake of decreasing the noise would hide much of the dynamics reflected in the spectral profiles.

The combination of high-resolution observations with simulations of much higher spatial resolution has demonstrated the need for high spatial resolution of $0 . ' 1$ and better, for high spectral resolution, for high temporal resolution, and for very low noise observation. Present detector technology will improve again and allow fast cadences of images, which are to be analysed with improved computer facilities. Perhaps the $1.5 \mathrm{~m}$ GREGOR telescope, which will be operative in the near future, will allow $0 . ' 1$ resolution in magnetograms. This will certainly be a new breakthrough in the research into the small-scale magnetism of the Sun. Still, it is important to have many photons to reduce noise. This is a typical case for solar telescopes of the $4 \mathrm{~m}$ class like EST and ATST.

Acknowledgements. We are greatly indebted to Robert Cameron for his invaluable and unselfish support with the numerical MHD simulations and for his encouragement with our English. NBG acknowledges financial support by the Deutsche Forchungsgemeinschaft through grant KN 152/31-1. L.Y.C. is grateful to the Max-Planck-Institut für Sonnensystemforschung, Katlenburg-Lindau, for a stipend of the International Max Planck Research School on "Physical Processes in the Solar System and Beyond". OO thanks for support by Deutsche Forschungsgemeinschaft through grant KN 152/29-3 and by the Institut für Astrophysik of the Georg-August-Universität Göttingen. The Vacuum Tower Telescope is operated by the Kiepenheuer-Institut für Sonnenphysik, Freiburg, at the Spanish Observatorio del Teide of the Instituto de Astrofísica de Canarias.

\section{References}

Abbett, W. P. 2007, ApJ, 665, 1469

Beckers, J. M., \& Schröter, E. H. 1968, Sol. Phys., 4, 142

Beck, C., Schmidt, W., Kentischer, T., \& Elmore, D. 2005a, A\&A, 437, 1159

Beck, C., Schlichenmaier, R., Collados, M., Bellot Rubio, L., \& Kentischer, T. 2005b, A\&A, 443, 1047

Bello González, N., \& Kneer, F. 2008, A\&A, 480, 265

Bello González, N., Okunev, O. V., Domínguez Cerdeña, I., Kneer, F., \& Puschmann, K. G. 2005, A\&A, 434, 317

Bello González, N., Kneer, F., \& Puschmann, K. G. 2007, in Modern Solar Facilities - Advanced Solar Science, ed. F. Kneer, K. G. Puschmann, \& A. D. Wittmann, Universitätsverlag Göttingen, 217

Bello González, N., Okunev, O., \& Kneer, F. 2008, A\&A, 490, L23

Bellot Rubio, L. R., Rodríguez Hidalgo, I., Collados, M., Khomenko, E., \& Ruiz Cobo, B. 2001, ApJ, 560, 1010

Bendlin, C., \& Volkmer, R. 1995, A\&AS, 112, 371

Bendlin, C., Volkmer, R., \& Kneer, F. 1992, A\&A, 257, 817

Bianda, M., Stenflo, J. O., \& Solanki, S. K. 1999, A\&A, 350, 1060

Carlsson, M., Stein, R. F., Nordlund, ̊., \& Scharmer, G. B. 2004, ApJ, 610, L137

Centeno, R., Socas-Navarro, H., Lites, B., et al. 2007, ApJ, 666, L137

Danilovic, S., and the MHD team at Max-Planck-Institut für Sonnensystemforschung, Katlenburg-Lindau, Germany 2008, private communication

de Boer, C. R. 1996, A\&AS, 120, 195

De Pontieu, B. 2002, ApJ, 569, 474

De Pontieu, B., Carlsson, M., Stein, R., et al. 2006, ApJ, 646, 1405

Domínguez Cerdeña, I., Sánchez Almeida, J., \& Kneer, F. 2003, A\&A, 407, 741 Domínguez Cerdeña, I., Sánchez Almeida, J., \& Kneer, F. 2006, ApJ, 646, 1421 Durney, B. R., De Young, D. S., \& Roxburgh, I. W. 1993, Sol. Phys., 145, 207

Eibe, M. T., Mein, P., Roudier, Th., \& Faurobert, M. 2001, A\&A, 371, 1128 Faurobert, M., Arnaud, J., Vigneau, J., \& Frisch, H. 2001, A\&A, 378, 627 Frutiger, C., Solanki, S. K., Fligge, M., \& Bruls, J. H. M. J. 2000, A\&A, 358, 1109

Grossmann-Doerth, U., Schüssler, M., \& Solanki, S. K. 1988, A\&A, 206, L37

Hasan, S. S., \& Schüssler, M. 1985, A\&A, 151, 69

Hirzberger, J., Bonet, J. A., Vázquez, M., \& Hanslmeier, A. 1999, ApJ, 527, 405

Janßen, K., Vögler, A., \& Kneer, F. 2003, A\&A, 409, 1127

Keller, C. U., \& von der Lühe, O. 1992, A\&A, 261, 321

Keller, C. U., Deubner, F.-L., Egger, U., Fleck, B., \& Povel, H. P. 1994, A\&A, 286,626

Khomenko, E. V., Collados, M., Solanki, S. K., Lagg, A., \& Trujillo Bueno, J. 2003, A\&A, 408, 1115

Khomenko, E. V., Martínez González, M. J., Collados, M., et al. 2005a, A\&A, 436, L27

Khomenko, E. V., Shelyag, S., Solanki, S. K., \& Vögler, A. 2005b, A\&A, 442, 1059

Kneer, F. 2007, in Modern Solar Facilities - Advanced Solar Science, ed. F. Kneer, K. G. Puschmann, \& A. D. Wittmann, Universitätsverlag Göttingen, 67

Koschinsky, M., Kneer, F., \& Hirzberger, J. 2001, A\&A, 365, 588 
Krieg, J., Wunnenberg, M., Kneer, F., Koschinsky, M., \& Ritter, C. 1999, A\&A, 343,983

Lin, H., \& Rimmele, T. 1999, ApJ, 514, 448

Lites, B. W., \& Socas-Navarro, H. 2004, ApJ, 613, 600

Lites, B. W., Leka, K. D., Skumanich, A., Martínez Pillet, V., \& Shimizu, T. 1996, ApJ, 460, 1019

Lites, B. W., Rutten, R. J., \& Berger, T. E. 1999, ApJ, 517, 1013

Lites, B. W., Kubo, M., Socas-Navarro, H., et al. 2008, ApJ, 672, 1237

Martínez González, M. J., Collados, M., Ruiz Cobo, B., \& Solanki, S. K. 2007a, A\&A, 469, L39

Martínez González, M. J., Asensio Ramos, A., López Ariste, A., \& Manso Sainz, R. 2007b, A\&A, 479, 229

Martínez González, M. J., Collados, M., Ruiz Cobo, B., \& Beck, C. 2008, A\&A, 477, 953

Martínez Pillet, V., Lites, B. W., \& Skumanich, A. 1997, ApJ, 474, 810

Nagata, S., Tsuneta, S., Suematsu, Y., et al. 2008, ApJ, 677, L145

Nordlund, Å., Spruit, H. C., Ludwig, H.-G., \& Trampedach, R. 1997, A\&A, 328, 229

Orozco Suárez, D., Bellot Rubio, L. R., del Toro Iniesta, J. C., et al. 2007, ApJ, 670, L61

Parker, E. N. 1979, Cosmical Magnetic Fields (Oxford: Clarendon)

Petrovay, K., \& Szakály, G. 1993, A\&A, 274, 543

Puschmann, K. G., Kneer, F., Seelemann, T., \& Wittmann, A. D. 2006, A\&A, 445,337

Rees, D. E., \& Semel, M. D. 1979, A\&A, 74, 1

Rezaei, R., Steiner, O., Wedemeyer-Böhm, S., Schlichenmaier, R., et al. 2007, A\&A, 476, L33

Sánchez Almeida, J., Collados, M., \& del Toro Iniesta, J. C. 1988, A\&A, 201, L37

Sánchez Almeida, J., Landi Degl'Innocenti, E., Martínez Pillet, V., \& Lites, B. W. 1996, ApJ, 466, 537
Schröter, E. H. 1957, ZAp, 41, 141

Schüssler, M., \& Vögler, A. 2008, A\&A, 481, L5

Semel, M. D. 1967, Ann. Astrophys., 30, 513

Sigwarth, M., Balasubramaniam, K. S., Knölker, M., \& Schmidt, W. 1999, A\&A, 349,941

Socas-Navarro, H., Martínez Pillet, V., \& Lites, B. W. 2004, ApJ, 611, 1139

Socas-Navarro, H., Borrero, J. M., Asensio Ramos, A., et al. 2008, ApJ, 674, 596

Solanki, S. K. 1987, Ph.D. Thesis, ETH Zürich

Stein, R. F., \& Nordlund, Å 2006, ApJ, 642, 1246

Steiner, O. 2000, Sol. Phys., 196, 245

Steiner, O. 2007, in Modern Solar Facilities - Advanced Solar Science, ed. F. Kneer, K. G. Puschmann, \& A. D. Wittmann, Universitätsverlag Göttingen, 321

Steiner, O., Grossmann-Doerth, U., Knölker, M., \& Schüssler, M. 1998, ApJ, 495,468

Steiner, O., Rezaei, R., Schaffenberger, W., \& Wedemeyer-Böhm, S. 2008, ApJ, 680, L85

Stenflo, J. O. 1973, Sol. Phys., 32, 41

Suematsu, Y., Tsuneta, S., Ichimoto, K., et al. 2008, Sol. Phys., 249, 197

Trujillo Bueno, J., Shchukina, N., \& Asensio Ramos, A. 2004, Nature, 430, 326 Vernazza, J. E., Avrett, E. H., Loeser, R. 1981, ApJS, 45, 635

Vögler, A. 2003, Ph.D. Thesis, Göttingen university

Vögler, A., Shelyag, S., Schüssler, M., et al. 2005, A\&A, 429, 335

Volkmer, R., Kneer, F., \& Bendlin, C. 1995, A\&A, 304, L1

von der Lühe, O. 1984, J. Opt. Soc. Am. A1, 510

von der Lühe, O., Soltau, D., Berkefeld, T., \& Schelenz, T. 2003, SPIE, 4853, 187

Weigelt, G. P. 1977, Optics Comm., 21, 55

Wunnenberg, M., Kneer, F., \& Hirzberger, J. 2002, A\&A, 395, L51 